Jan Burczak

\title{
Almost everywhere Hölder continuity of gradients to non-diagonal parabolic systems
}

\author{
Received: 30 March 2013 / Revised: 27 July 2013 \\ Published online: 23 October 2013
}

\begin{abstract}
We present a local almost everywhere $C^{1, \alpha}$-regularity result for a general class of p-nonlinear non-diagonal parabolic systems. The main part of the considered systems depends on space-time variable, solution and symmetric part of the gradient of solution. To obtain our result, we adapt for the symmetric-gradient case techniques developed for the full-gradient case by Duzaar, Mingione and coauthors.
\end{abstract}

\section{Introduction}

The problem of local Hölder continuity of gradients for the evolutionary $p$-Laplace system has been resolved in a series of papers by DiBenedetto and coauthors, summed up in a monograph [7], with crucial earlier (stationary) contributions of Uhlenbeck [23], Tolksdorf [22] and the Russian school. From the perspective of mathematical physics, it is interesting to replace $\nabla u$ by its symmetric part $\mathbb{D} u=$ $\left(\nabla u+\nabla^{T} u\right) / 2$; then such a symmetric $p$-Laplace system is a simplification of the hydrodynamic model of a non-Newtonian flow (referred to as $p$-Navier-Stokes in the following). In fact, for $p>11 / 5$, the generalization from a $p$-Stokes system to the respective $p$-Navier-Stokes is not essential from the perspective of regularity theory (compare [16]).

It turns out that the amendment from $\nabla u$ to $\mathbb{D} u$ in a system of the $p$-Laplace type, a supposedly harmless one, diminishes dramatically our understanding of $C^{1, \alpha}$-regularity of such system. The main difficulty lies in the fact that most of the methods, which are successful in the full gradient case, depend essentially on a pointwise structure, peculiar only to full-gradient $p$-Laplace system. In this article we show, however, that the $p$-caloric approximation approach can still be used to obtain almost everywhere regularity. We consider parabolic systems of the following type

J. Burczak $(\varangle)$ : Institute of Mathematics, Polish Academy of Sciences,

Śniadeckich 8, 00-950 Warsaw, Poland

e-mail: jb@impan.pl

Mathematics Subject Classification (2000): MSC 35K55, MSC 35B65, MSC 35K92

Supported by the International Ph.D. Projects Programme of Foundation for Polish Science within the Innovative Economy Operational Programme 2007-2013. Partially supported by the National Science Centre (NCN) grant no. 2011/01/N/ST1/05411. 


$$
u_{, t}-\operatorname{div} A(z, u, \mathbb{D} u)=0
$$

the prototype of which is the following symmetric p-Laplace system with safety-1

$$
u_{, t}-\operatorname{div}\left[\left(1+|\mathbb{D} u|^{2}\right)^{\frac{p-2}{2}} \mathbb{D} u\right]=0
$$

Let us provide the reader with a short account of relevant known results. In [17] an extensive short-time maximal regularity theory in Sobolev-Slobodeckii spaces for $p$-Navier-Stokes is presented by Prüss and Bothe. However, not much is known on the global-in-time $C^{1, \alpha}$-regularity of such systems in arbitrary dimension $d$ (or at least for physically plausible $d \geq 3$ ), even for the prototype case. It is worth mentioning that for $p \in(12 / 5 ; 10 / 3)$ Ladyzhenskaya and Seregin have shown in [19] an almost-everywhere regularity result for the complete three-dimensional hydrodynamic system. One can also easily see from the theory developed in [13] by Kaplický, Málek, Stará for the two-dimensional $p$-Navier-Stokes that system (1.2) and some of its generalizations enjoy $C^{1, \alpha}$-regularity in the case of twodimensions. The current research status is a little clearer in the case of stationary simplifications. There is a well developed $C^{1, \alpha}$-theory for the stationary $p$-Laplace and $p$-Navier-Stokes systems with $p<2$ by Beirão da Veiga and collaborators (see [2] and references therein). In the case of $p \geq 2$, one can refer to [1], where Apushkinskaya, Bildhauer and Fuchs obtain partial $C^{1, \alpha}$-regularity for three-dimensional $p$-Stokes and full $C^{1, \alpha}$-regularity in the two-dimensional case.

In this paper we follow the theory based on $p$-caloric approximations, which has been developed for the full-gradient case and very general main parts in [9], [10] by Duzaar, Mingione and coauthors. We apply their ideas for the symmetricgradient case. At some points we could have merely quoted the respective results from [10]; instead, for reader's convenience, most of the proofs are presented with concern for the clarity of exposition.

However the $p$-caloric approach seems to be very well-suited also for our symmetric-gradient case, let us emphasize that our result seems to be new not only for general system (1.1), but even for its prototype (1.2). As a byproduct, which may be interesting by itself, we obtain also a Campanato-type theory for linear parabolic systems satisfying Legendre-Hadamard conditions, for which we couldn't find a satisfactory reference.

\section{Notation and statement of the result}

The expression $A=: B$ means that $A$ defines $B$. Denote a space-time point $z=$ $(x, t) \in \Omega \times(-T, 0)=: Q$, where $\Omega \subset \mathbb{R}^{d}$. As we develop a local interior regularity theory, any further assumptions on domain $Q$ are unnecessary. $B_{r}(x), Q_{r}(z)$ denote, respectively, the ball with the radius $r$ centered at a point $x$ and the parabolic cylinder $B_{r}(x) \times\left(t-r^{2}, t\right) . \partial_{\Gamma} \tilde{Q}$ denotes parabolic boundary of cylinder $\tilde{Q}$.

For a tensor $\xi \in \mathbb{R}^{d \times d}$ denote its symmetric part by $\xi^{s}:=\left(\xi+\xi^{T}\right) / 2$. For any matrix $M \in \mathbb{R}^{d^{2} \times d^{2}}$ denote its coefficients by $M_{k l}^{i j}$; its action on tensor $\xi$ with coefficients $\xi_{k l}$ is $M_{k l}^{i j} \xi_{k l}$ (here and further on we use the summation convention). Sym $m^{d \times d}$ denotes set of $d \times d$ symmetric tensors. 
We use standard notation for function spaces; $L^{p}\left(\tau, t ; W^{1, p}\left(B_{\varrho}(z)\right)\right)$ will be sometimes abbreviated to $L^{p}\left(W^{1, p}\right)$, when there is no danger of confusion regarding underlying cylinder.

Let us emphasize that constants denoted by $C$ may change from line to line of estimates and are bigger than 1. If a more careful control over a constant is needed, we denote their dependence on certain parameters writing $C$ (parameter) and generally suppress marking their dependence on irrelevant parameters; such constants may also vary. For clarity we also use some fixed constants, which we denote by $C_{\text {subscript }}$.

Now let us present a list of assumptions for the studied generalization (1.1) to (1.2). For any tensors $\xi, \eta \in \mathbb{R}^{d \times d}$

- main part $A$ satisfies properties of

$$
\begin{aligned}
A\left(z, u, \xi^{s}\right) \xi^{s} & \geq \lambda\left|\xi^{s}\right|^{p} \quad \text { (being strongly elliptic), } \\
A\left(z, u, \xi^{s}\right) \eta & \geq A\left(z, u, \xi^{s}\right) \eta^{s} \quad \text { (being weakly symmetrizing), } \\
|A(z, u, q)| & \leq C\left(1+|q|^{p-1}\right) \text { (having } p-1 \text { growth), } \\
|A(z, u, q)-A(\tilde{z}, \tilde{u}, q)| & \leq C \theta(z, \tilde{z}, u, \tilde{u})\left(1+|q|^{p-1}\right)
\end{aligned}
$$

where $\beta \in(0,1), \theta=\min \left[1, K(|u|+|\tilde{u}|)\left(d_{2}(z-\tilde{z})+|u-\tilde{u}|^{\beta}\right)\right]$ with a nondecreasing real function $K: \mathbb{R}_{+} \rightarrow[1, \infty)$ and the parabolic metric $d_{2}(z-$ $\left.z_{0}\right):=\left|x-x_{0}\right|+\left|t-t_{0}\right|^{\frac{1}{2}}$

- whereas $\frac{\partial A}{\partial q}(z, u, q)=\frac{\partial A}{\partial q}(\cdot)$

$$
\begin{aligned}
& \text { is Legendre-Hadamard elliptic } \frac{\partial A}{\partial q}(\cdot) \xi^{s} \cdot \xi^{s} \geq \lambda\left(1+|q|^{2}\right)^{\frac{p-2}{2}}\left|\xi^{s}\right|^{2}, \\
& \text { is strongly symmetrizing }\left(\frac{\partial A}{\partial q}(\cdot)\right)_{k l}^{i j}=\left(\frac{\partial A}{\partial q}(\cdot)\right)_{i j}^{k l}=\left(\frac{\partial A}{\partial q}(\cdot)\right)_{l k}^{j i},
\end{aligned}
$$

grows in a general way $|u|+|q| \leq M \Longrightarrow\left|\frac{\partial A}{\partial q}(z, u, q)\right| \leq C_{(2.2 \mathrm{c})}(M)$,

$$
\begin{aligned}
& \text { is continuous } \begin{aligned}
|u|+|q|+|u-\tilde{u}| & +|q-\tilde{q}| \leq M \\
\Longrightarrow\left|\frac{\partial A}{\partial q}(z, u, q)-\frac{\partial A}{\partial \tilde{q}}(\tilde{z}, \tilde{u}, \tilde{q})\right| \leq & C(M) \omega\left(M, d_{2}^{2}(z-\tilde{z})\right. \\
& \left.+|u-\tilde{u}|^{p}+|q-\tilde{q}|^{p}\right),
\end{aligned}
\end{aligned}
$$

and the local modulus of continuity $\omega$ satisfies: $\omega(\cdot, s), \omega(t, \cdot)$ are nondecreasing, $\omega(t, 0)=0$ and $\omega(t, \cdot)$ is continuous at zero, $\omega^{p}(t, \cdot)$ is concave.

Remark 2.1. Observe that property $(2.2 \mathrm{~d})$ is indeed merely continuity and that (2.2b) implies that $\frac{\partial A}{\partial q}$ is weakly symmetrizing, i.e.

$$
\frac{\partial A}{\partial q}(z, u, q) \xi^{s} \cdot \eta=\frac{\partial A}{\partial q}(z, u, q) \xi^{s} \eta^{s}
$$

The main result reads. 
Theorem 2.1. Any weak solution $u \in C\left(-T, 0 ; L^{2}(\Omega)\right) \cap L^{p}\left(-T, 0 ; W^{1, p}(\Omega)\right)$ to the system (1.1) with $p \geq 2$ and structure (2.1a-2.2d) has a.e. Hölder continuous gradients and the solution itself is also a.e. Hölder continuous.

More precisely, there is an open set $\tilde{Q}$ of full Lebesgue measure containing

$$
\left\{z \in Q: \liminf _{\varrho \rightarrow 0} f_{Q_{\varrho}(z)}\left|\nabla u-(\nabla u)_{z}\right|^{p}=0 \wedge \limsup _{\varrho \rightarrow 0}\left|(u)_{z, \varrho}\right|+\left|(\nabla u)_{z, \varrho}\right|<+\infty\right\}
$$

for which

$$
\nabla u \in C^{\beta, \frac{\beta}{2}}(\tilde{Q}), \quad u \in C^{1, \frac{1}{2}}(\tilde{Q}),
$$

where $\beta$ comes from (2.1d).

Let us repeat that, to our best knowledge, even for the prototype system (1.2) this result is new.

\section{Outline of the paper}

The rest of the article is devoted to the proof of Theorem 2.1. For traceability, let us first present the outline of the paper.

- In Sect. 4 auxiliary lemmas are gathered. This includes a Campanato-type regularity theory for linear parabolic systems satisfying Legendre-Hadamard conditions, see Lemma 4.4, and the symmetric caloric approximation lemma-Lemma 4.5. The latter states that every function which is close in a certain weak sense to a solution of a linear parabolic system, with its main part dependent on symmetric gradient, is indeed close to a solution of a linear parabolic system in a strong sense.

- Section 5 is devoted to showing, by means of linearization and Caccioppoli inequality, that an appropriately rescaled weak solution to (1.1) satisfies locally certain inequalities that resemble assumptions of the caloric approximation lemma. This is done via Lemmas 5.2, 5.3 and summed up in Corollary 5.4.

- Section 6 combines results of the previous sections and gives the proof of Theorem 2.1. Namely, thanks to Corollary 5.4, around points which satisfy certain regularity assumptions, one can use caloric approximation for (rescaled) solution of (1.1), which thanks to the regularity of linear systems gives proper shrinking of excess energies (Lemma 6.1). This yields, by iteration, the Hölder continuity of gradients (Lemma 6.3).

Only the crucial results are proved directly after their statements; for the sake of clarity, the remaining proofs are transferred to the Sect. 7-Appendix. 


\section{Useful auxiliary results}

This section begins with a Simon-type compactness result for parabolic spaces, which can be found as Theorem 2.5 in [10].

Lemma 4.1. Take $p \in(1, \infty)$, three Banach spaces $X \subset \subset Y \subset Z$ and a sequence $g_{k}$, which is uniformly bounded in $L^{p}(-T, 0 ; X)$ and satisfies

$$
\underset{\varepsilon>0}{\forall} \underset{h^{\prime}>0}{\exists} \underset{h \in\left(0, h^{\prime}\right]}{\forall} \int_{-T}^{-h}\left|g_{k}(\cdot, t+h)-g_{k}(\cdot, t)\right|_{Z}^{p} d t \leq \varepsilon
$$

then $g_{k}$ contains a subsequence convergent in the space $L^{p}(-T, 0 ; Y)$.

The next result collects properties needed to perform analysis of excess energies (see Definition 5.1). For proof see [10] Lemma 2.1; the last inequality can be found in proof of Lemma 4.8 there. Compare also [15].

Lemma 4.2. Let $u \in L^{s}\left(Q\left(z_{0}\right)\right), s \geq 2$, where $z_{0}=\left(x_{0}, t_{0}\right)$. There is the unique minimizer $l_{z_{0}, \varrho}^{(s)}(x)$ to $\int_{Q_{\varrho}\left(z_{0}\right)}|u-l|^{s}$ among affine, time-independent functions $l$; moreover

$$
l_{z_{0}, \varrho}^{(2)}(x)=(u)_{z_{0}, \varrho}+\left[\frac{d+2}{\varrho^{2}} f_{Q_{\varrho}\left(z_{0}\right)} u(x, t) \otimes\left(x-x_{0}\right) d x d t\right]\left(x-x_{0}\right)
$$

the linear part $\nabla l_{z_{0}, \varrho}^{(2)}$ of which is close to $(\nabla u)_{z_{0}, \varrho}$

$$
\left|\nabla l_{z_{0}, \varrho}^{(2)}-(\nabla u)_{z_{0}, \varrho}\right|^{2} \leq \frac{d(d+2)}{\varrho^{2}} \int_{Q_{\varrho}\left(z_{0}\right)}\left|u-(u)_{z_{0}, \varrho}-(\nabla u)_{z_{0}, \varrho}\left(x-x_{0}\right)\right|^{2}
$$

and shrinks as follows

$$
\left|\nabla l_{z_{0}, \theta \varrho}^{(2)}-\nabla l_{z_{0}, \varrho}^{(2)}\right|^{2} \leq \frac{d(d+2)}{(\theta \varrho)^{2}} f_{Q_{\varrho}\left(z_{0}\right)}\left|u-l_{\varrho}^{(2)}\right|^{2}
$$

For the minimizer in the case of general $s \geq 2$ holds

$$
f_{Q_{\varrho}\left(z_{0}\right)}\left|l_{\varrho}^{(2)}-l_{\varrho}^{(s)}\right|^{s} \leq C_{(4.5)}(d, s) \underset{Q_{\varrho}\left(z_{0}\right)}{f}\left|u-l_{\varrho}^{(s)}\right|^{s}
$$

Subsequently let us state the Korn's inequality. For hints for proof, see the Appendix. 
Lemma 4.3. (Korn's inequality) For $u \in W^{1, p}\left(B_{r}(x)\right)$ following inequalities hold with $C_{K o r_{p}}$ independent on radius of $B_{r}(x)$

$$
\begin{array}{r}
C_{K_{\text {or }}}\left[\int_{B_{r}(x)} r^{-p}|u|^{p}+|\mathbb{D} u|^{p}\right] \geq \int_{B_{r}(x)}|\nabla u|^{p}, \\
C_{K_{\text {or }}} \int_{B_{r}(x)}|\mathbb{D} u-(\mathbb{D} u)|^{2} \geq \int_{B_{r}(x)}|\nabla u-(\nabla u)|^{2} \geq \int_{B_{r}(x)}|\mathbb{D} u-(\mathbb{D} u)|^{2}
\end{array}
$$

Next lemma, which may be of independent interest, collects needed results on linear parabolic systems with main part depending on symmetric gradient. Recall that $A M$ denotes constant coefficient matrix $A$ with elements $a_{k l}^{i j}$ acting on tensor $M$ with elements $m_{k l}$, i.e. $A M=a_{k l}^{i j} m_{k l}$. Again we refer to the Appendix for the proof.

Lemma 4.4. (Campanato-type regularity theory for linear parabolic systems satisfying Legendre-Hadamard conditions) Let $u \in L^{2}\left(-T, 0 ; W^{1,2}(\Omega)\right)$ be a local solution to $u_{, t}-\operatorname{div} A \mathbb{D} u=0$, i.e. let it satisfy

$$
\int_{\Omega_{T}} u \varphi_{, t}-A \mathbb{D} u \mathbb{D} \phi=0 \quad \underset{\varphi \in C_{0}^{\infty}\left(\Omega_{T}\right)}{\forall}
$$

where for constant coefficient matrix A holds:

$$
\begin{gathered}
a_{k l}^{i j}=a_{i j}^{k l}=a_{l k}^{j i} \\
a_{k l}^{i j} \xi_{l} \xi_{j} \eta^{k} \eta^{i}+a_{k l}^{i j} \xi_{k} \xi_{j} \eta^{l} \eta^{i} \geq \lambda|\eta|^{2}|\xi|^{2} \\
\underset{\eta, \xi \in \mathbb{R}^{d}}{\forall} \\
A \xi^{s} \xi^{s} \geq \lambda\left|\xi^{s}\right|^{2} \\
\underset{\xi \in \mathbb{R}^{d \times d}}{\forall}
\end{gathered}
$$

then $u$ is locally smooth and satisfies for any $p, q \in[1, \infty], \varrho \leq r / 2$, arbitrary $\tilde{z}_{0} \in Q_{\varrho}$

$$
\begin{gathered}
{\left[f_{Q_{\varrho}}\left|u^{(m)}\right|^{q}\right]^{\frac{1}{q}} \leq C_{(4.9)}(\lambda,|A|, m, d, p, q) r^{-2 m}\left[f_{Q_{r}}|u|^{p}\right]^{\frac{1}{p}}, \text { (4.9) }} \\
{\left[f_{Q_{\varrho}}\left|u^{(m)}-u^{(m)}\left(\tilde{z}_{0}\right)\right|^{q}\right]^{\frac{1}{q}} \leq C_{(4.10)}(\lambda,|A|, m, d, p, q) r^{-2 m}\left(\frac{\varrho}{r}\right)\left[f_{Q_{r}}|u|^{p}\right]^{\frac{1}{p}},}
\end{gathered}
$$


$\left[f_{Q_{\varrho}}\left|u^{(m)}-\left(u^{(m)}\right)_{Q_{\varrho}}\right|^{q}\right]^{\frac{1}{q}} \leq C_{(4.11)}(\lambda,|A|, m, d, p, q) r^{-2 m}\left(\frac{\varrho}{r}\right)\left[f_{Q_{r}}|u|^{p}\right]^{\frac{1}{p}}$,

where $u^{(m)}$ denotes either $\nabla^{(2 m)} u$ or $\partial_{t}^{(m)} u$ and $|a|^{s}=\sum_{n=1}^{N}\left|a_{n}\right|^{s}$ for $a \in \mathbb{R}^{N}$.

As outlined in the introduction, we end this section by stating a local result which says that a function, which is approximately solving a certain linear system in a weak sense (such function is called approximatively weakly symmetrical caloric in the following), is indeed close to some solution to this system in an appropriate strong $L^{2}-L^{p}$ sense. The idea can be traced back to L. Simon, see [20]. The proof, up to few technicalities connected with symmetric gradient, is identical with its counterpart in [10] and can be found in the Appendix. We work now with fixed $p \geq 2$ and cylinder $Q_{\varrho}\left(z_{0}\right)$ (therefore they does not appear as parameters). Let us introduce some definitions.

Definition 4.1. $S(\lambda, \Lambda)$ denotes the set of elliptic bilinear forms, which have the properties of being symmetrizing and $\lambda$-elliptic and $\Lambda$-bounded. Precisely:

$$
\begin{aligned}
& S(\lambda, \Lambda) \\
& :=\left\{A: \mathbb{R}^{d^{2}} \times \mathbb{R}^{d^{2}} \rightarrow \mathbb{R}, \text { bilinear, } a_{k l}^{i j}=a_{l k}^{j i}, \lambda\left|\xi^{s}\right|^{2} \leq A \xi^{s} \xi^{s},|A| \leq \Lambda \underset{\xi, \in \mathbb{R}^{d^{2}}}{\forall}\right.
\end{aligned}
$$

Observe that A is weakly symmetrizing, as $a_{k l}^{i j}=a_{l k}^{j i}$ implies $A \xi^{s} \eta=A \xi^{s} \eta^{s}$.

In the following two definitions $\delta>0, \gamma \geq 0$ are number parameters.

Definition 4.2. Set $H(r ; \delta, A, \gamma)$ of approximatively weakly symmetrical caloric functions consists of elements of $L^{p}\left(t_{0}-r^{2}, t_{0} ; W^{1, p}\left(B_{r}\left(z_{0}\right)\right)\right.$ that satisfy for all $\varphi \in C_{0}^{\infty}\left(Q_{r}\left(z_{0}\right)\right)$

$$
\begin{aligned}
f_{Q_{r}\left(z_{0}\right)}\left|\frac{f}{r}\right|^{2}+|\nabla f|^{2}+\gamma^{p-2}\left[\left|\frac{f}{r}\right|^{p}+|\nabla f|^{p}\right] & \leq 1, \\
& \left|f_{Q_{r}\left(z_{0}\right)} f \varphi_{, t}-A(\mathbb{D} f, \mathbb{D} \varphi)\right| \leq \delta \cdot|\mathbb{D} \varphi|_{L^{\infty}}
\end{aligned}
$$

Definition 4.3. Set $H(r ; A, \gamma)$ of caloric symmetrical functions constitute $f \in$ $L^{p}\left(t_{0}-r^{2}, t_{0} ; W^{1, p}\left(B_{r}\left(z_{0}\right)\right)\right.$ such that for all $\varphi \in C_{0}^{\infty}\left(Q_{r}\left(z_{0}\right)\right)$

$$
\begin{aligned}
f_{Q_{r}\left(z_{0}\right)}\left|\frac{f}{r}\right|^{2}+|\nabla f|^{2}+\gamma^{p-2}\left[\left|\frac{f}{r}\right|^{p}+|\nabla f|^{p}\right] & \leq 2^{d+3}, \\
f_{Q_{r}\left(z_{0}\right)} f \varphi_{, t}-A(\mathbb{D} f, \mathbb{D} \varphi) & =0
\end{aligned}
$$


Lemma 4.5. (symmetric caloric approximation lemma) Take $p \geq 2$. Fix positive $\varepsilon, \lambda, \Lambda$. Then there exists $\delta \in(0,1)$, common for: all $A \in S(\lambda, \Lambda)$ and $\gamma \in[0,1]$, such that the following implication holds

$$
f \in H(\varrho ; \delta, A, \gamma) \Rightarrow \underset{h \in H(\varrho / 2 ; A, \gamma)}{\exists} f_{Q_{\varrho / 2}\left(z_{0}\right)}^{\exists}\left|\frac{h-f}{\varrho / 2}\right|^{2}+\gamma^{p-2}\left|\frac{h-f}{\varrho / 2}\right|^{p} \leq \varepsilon
$$

\section{Local estimates}

Let us emphasize that in this section the dependence of constants $C$ on irrelevant parameters is suppressed. All cylinders $Q_{z_{0}}(\varrho) \subset Q$ used below have $\varrho \leq 1$. First let us define local excess energies

\section{Definition 5.1.}

$$
\phi_{p, z_{0}, l}(\varrho):=f_{Q_{z_{0}}(\varrho)}|\mathbb{D} u-\mathbb{D} l|^{p}, \quad \psi_{p, z_{0}, l}(\varrho):=f_{Q_{z_{0}}(\varrho)}\left|\frac{u-l}{\varrho}\right|^{p}
$$

For briefness, using the energies defined above we often drop certain parameters, writing for example $\phi_{p}(\varrho), \psi_{p}(\varrho)$. First we state an auxiliary algebraic lemma needed for the estimates of this section.

Lemma 5.1. (Algebraic inequalities) Assume that for a matrix A condition (2.1d) is valid. Then, for any: $z \in Q_{z_{0}}(\varrho), u \in \mathbb{R}^{d}, P \in S m^{d \times d}$, affine function $l(x)$ the following inequalities hold

$$
\begin{gathered}
\left|A\left(z_{0}, l\left(z_{0}\right), P\right)-A(z, u, P)\right| \leq C\left(\left|l\left(z_{0}\right)\right|+|\nabla l|\right) \varrho^{\beta}\left[1+|P-\mathbb{D} l|^{p}+\left|\frac{u-l}{\varrho}\right|^{p}\right] \\
\left|A\left(z_{0}, l\left(z_{0}\right), \mathbb{D} l\right)-A(z, u, \mathbb{D} l)\right| \leq C\left(\left|l\left(z_{0}\right)\right|+|\nabla l|\right) \varrho^{\beta}\left[1+\left|\frac{u-l}{\varrho}\right|^{\beta}\right]
\end{gathered}
$$

If, additionally, A satisfies (2.1c), (2.2c), then it also holds

$$
\begin{aligned}
& |A(z, u, P)-A(z, u, \mathbb{D} l)| \\
& \quad \leq C\left(\left|l\left(z_{0}\right)\right|+|\nabla l|\right)\left(|u-l|^{\beta}+|u-l|+|P-\mathbb{D} l|^{p-1}+|P-\mathbb{D} l|\right) \\
& \left|A(z, u, P)-A\left(z_{0}, l\left(z_{0}\right), \mathbb{D} l\right)\right| \leq C\left(\left|l\left(z_{0}\right)\right|+|\nabla l|\right)\left(1+|P|^{p-1}\right)
\end{aligned}
$$

Proof of this lemma has been shifted to appendix.

Lemma 5.2. (Linearization) Take $p \geq 2$ and $u \in L^{p}\left(-T, 0 ; W^{1, p}(\Omega)\right)$ solving (1.1) with structure: $(2.1 b-2.1 d),(2.2 c),(2.2 d)$. For any $M>0, Q_{\varrho}\left(z_{0}\right), \varphi \in$ $C_{0}^{\infty}\left(Q_{\varrho}\left(z_{0}\right)\right)$, affine function $l(z)=l(x)$ such that $\left|l\left(z_{0}\right)\right|+|\nabla l| \leq M$ we have: 


$$
\begin{aligned}
& \left|\int_{Q_{\varrho}\left(z_{0}\right)}(u-l) \varphi_{, t}-\frac{\partial A}{\partial q}\left(z_{0}, l\left(z_{0}\right), \mathbb{D} l\right)(\mathbb{D} u-\mathbb{D} l) \mathbb{D} \varphi\right| \\
& \leq C_{l i n}(M)\left[\omega\left(M+1, \phi_{p}\right) \phi_{2}^{\frac{1}{2}}+\phi_{p}+\psi_{p}+\varrho^{\beta}\right] \sup _{Q_{\varrho}\left(z_{0}\right)}|\mathbb{D} \varphi|
\end{aligned}
$$

Proof. Use time-independence of $l$ to get from weak formulation of (1.1) that for any $\varphi \in C_{0}^{\infty}\left(Q_{\varrho}\left(z_{0}\right)\right)$ holds

$$
0=\int_{Q_{\varrho}\left(z_{0}\right)}(u-l) \varphi_{, t}-A(z, u, \mathbb{D} u) \mathbb{D} \varphi
$$

which by adding and subtracting certain terms yields

$$
\begin{aligned}
f_{Q_{\varrho}\left(z_{0}\right)} & (u-l) \varphi, t-\frac{\partial A}{\partial q}\left(z_{0}, l\left(z_{0}\right), \mathbb{D} l\right)(\mathbb{D} u-\mathbb{D} l) \mathbb{D} \varphi \\
= & \int_{Q_{\varrho}\left(z_{0}\right)}\left[A(z, u, \mathbb{D} u)-A\left(z_{0}, l\left(z_{0}\right), \mathbb{D} u\right)\right] \mathbb{D} \varphi \\
& +f_{Q_{\varrho}\left(z_{0}\right)}\left[A\left(z_{0}, l\left(z_{0}\right), \mathbb{D} u\right)-\frac{\partial A}{\partial q}\left(z_{0}, l\left(z_{0}\right), \mathbb{D} l\right) \cdot(\mathbb{D} u-\mathbb{D} l)\right] \mathbb{D} \varphi
\end{aligned}
$$

We need to estimate the right-hand-side of (5.7). First, estimate second integral on the r.h.s. of (5.7) with respect to the splitting of $Q_{\varrho}\left(z_{0}\right)$ into

$$
\begin{aligned}
& Q_{\varrho}^{s}=Q_{\varrho}\left(z_{0}\right) \cap\{|\mathbb{D} u-\mathbb{D} l| \leq 1\} \\
& Q_{\varrho}^{b}=Q_{\varrho}\left(z_{0}\right) \cap\{|\mathbb{D} u-\mathbb{D} l|>1\}
\end{aligned}
$$

i.e.

$$
\frac{1}{\left|Q_{\varrho}\right|} \int_{Q_{\varrho}^{s}}\left[A\left(z_{0}, l\left(z_{0}\right), \mathbb{D} u\right)-\frac{\partial A}{\partial q}\left(z_{0}, l\left(z_{0}\right), \mathbb{D} l\right) \cdot(\mathbb{D} u-\mathbb{D} l)\right] \mathbb{D} \varphi
$$

and

$$
\frac{1}{\left|Q_{\varrho}\right|} \int_{Q_{\varrho}^{b}}\left[A\left(z_{0}, l\left(z_{0}\right), \mathbb{D} u\right)-\frac{\partial A}{\partial q}\left(z_{0}, l\left(z_{0}\right), \mathbb{D} l\right) \cdot(\mathbb{D} u-\mathbb{D} l)\right] \mathbb{D} \varphi
$$

without loss of generality we may assume that neither $Q_{\varrho}^{s}$ nor $Q_{\varrho}^{b}$ is empty. Since $A(\cdot, 0)=f_{Q_{\varrho}^{s}} A\left(z_{0}, l\left(z_{0}\right), \mathbb{D} l\right) \mathbb{D} \varphi=0$ one infers that $(5.9)$ is

$$
\begin{aligned}
\frac{1}{\left|Q_{\varrho}\right|} \int_{Q_{\varrho}^{s}} \int_{0}^{1} & {\left[\frac{\partial A}{\partial q}\left(z_{0}, l\left(z_{0}\right), \mathbb{D} l+\tau(\mathbb{D} u-\mathbb{D} l)\right)\right.} \\
& \left.-\frac{\partial A}{\partial q}\left(z_{0}, l\left(z_{0}\right), \mathbb{D} l\right)\right](\mathbb{D} u-\mathbb{D} l) \mathbb{D} \varphi d \tau .
\end{aligned}
$$


On $Q_{\varrho}^{s}$ holds $\left|l\left(z_{0}\right)\right|+|\mathbb{D} l|+|\mathbb{D} u-\mathbb{D} l| \leq M+1$ in view of assumptions on $l$, so by $(2.2 \mathrm{~d})$

$$
\begin{aligned}
& \left|\int_{0}^{1}\left[\frac{\partial A}{\partial q}\left(z_{0}, l\left(z_{0}\right), \mathbb{D} l+\tau(\mathbb{D} u-\mathbb{D} l)\right)-\frac{\partial A}{\partial q}\left(z_{0}, l\left(z_{0}\right), \mathbb{D} l\right)\right](\mathbb{D} u-\mathbb{D} l) \mathbb{D} \varphi d \tau\right| \\
& \quad \leq C(M) \omega\left(M+1,|\mathbb{D} u-\mathbb{D} l|^{p}\right)|\mathbb{D} u-\mathbb{D} l||\mathbb{D} \varphi|
\end{aligned}
$$

Merging (5.11) and (5.12) one has

$$
\begin{aligned}
& \frac{1}{\left|Q_{\varrho}\right|} \int_{Q_{\varrho}^{s}} \int_{0}^{1}\left[\frac{\partial A}{\partial q}\left(z_{0}, l\left(z_{0}\right), \mathbb{D} l+\tau(\mathbb{D} u-\mathbb{D} l)\right)-\frac{\partial A}{\partial q}\left(z_{0}, l\left(z_{0}\right), \mathbb{D} l\right)\right](\mathbb{D} u-\mathbb{D} l) \mathbb{D} \varphi d \tau \\
& \leq C(M)\left[f_{Q_{\varrho}\left(z_{0}\right)} \omega^{p}\left(M+1,|\mathbb{D} u-\mathbb{D} l|^{p}\right)\right]^{1 / p}\left[f_{Q_{\varrho}\left(z_{0}\right)}|\mathbb{D} u-\mathbb{D} l|^{p^{\prime}}\right]^{1 / p^{\prime}} \\
& \quad \times \sup _{Q_{\varrho}\left(z_{0}\right)}|\mathbb{D} \varphi| \leq C(M) \omega\left(M+1, \phi_{p}\right) \phi_{p^{\prime}}^{1 / p^{\prime}} \sup _{Q_{\varrho}\left(z_{0}\right)}|\mathbb{D} \varphi| \\
& \leq C(M) \omega\left(M+1, \phi_{p}\right) \phi_{2}^{1 / 2} \sup _{Q_{\varrho}\left(z_{0}\right)}|\mathbb{D} \varphi|
\end{aligned}
$$

where the last two inequalities hold by concavity of $\omega^{p}(t, \cdot)$ and $p \geq 2$. Therefore we can estimate (5.9) as follows

$$
\begin{gathered}
\frac{1}{\left|Q_{\varrho}\right|} \int_{Q_{\varrho}^{s}}\left[A\left(z_{0}, l\left(z_{0}\right), \mathbb{D} u\right)-\frac{\partial A}{\partial q}\left(z_{0}, l\left(z_{0}\right), \mathbb{D} l\right) \cdot(\mathbb{D} u-\mathbb{D} l)\right] \mathbb{D} \varphi \\
\leq C(M) \omega\left(M+1, \phi_{p}\right) \phi_{p}^{1 / p} \sup _{Q_{\varrho}\left(z_{0}\right)}|\mathbb{D} \varphi|
\end{gathered}
$$

Consider now (nonempty) $Q_{\varrho}^{b}$. One has for any $s>1$

$$
\frac{\left|Q_{\varrho}^{b}\right|}{\left|Q_{\varrho}\right|} \leq f_{Q_{\varrho}}|\mathbb{D} u-\mathbb{D} l|^{s}
$$

because

$$
\begin{aligned}
\left|Q_{\varrho}^{b}\right| \leq \int_{Q_{\varrho}^{b}}|\mathbb{D} u-\mathbb{D} l| & \leq\left(\int_{Q_{\varrho}^{b}}|\mathbb{D} u-\mathbb{D} l|^{s}\right)^{1 / s}\left|Q_{\varrho}^{b}\right|^{1 / s^{\prime}} \\
& \leq\left(f_{Q_{\varrho}}|\mathbb{D} u-\mathbb{D} l|^{s}\right)^{1 / s}\left|Q_{\varrho}^{b}\right|^{1 / s^{\prime}}\left|Q_{\varrho}\right|^{1 / s}
\end{aligned}
$$


From $|\mathbb{D} l|+\left|l\left(z_{0}\right)\right| \leq M$ and (2.1c), (2.2c) we estimate (5.10)

$$
\begin{aligned}
\frac{1}{\left|Q_{\varrho}\right|} \int_{Q_{\varrho}^{b}} & {\left[A\left(z_{0}, l\left(z_{0}\right), \mathbb{D} u\right)-\frac{\partial A}{\partial q}\left(z_{0}, l\left(z_{0}\right), \mathbb{D} l\right)(\mathbb{D} u-\mathbb{D} l)\right] \mathbb{D} \varphi } \\
\leq & \sup _{Q_{\varrho}\left(z_{0}\right)}|\mathbb{D} \varphi| \frac{C(M)}{\left|Q_{\varrho}\right|} \int_{Q_{\varrho}^{b}} 1+|\mathbb{D} u|^{p-1}+|\mathbb{D} u-\mathbb{D} l| \\
\leq & \sup _{Q_{\varrho}\left(z_{0}\right)}|\mathbb{D} \varphi| \frac{C(M)}{\left|Q_{\varrho}\right|} \int_{Q_{\varrho}^{b}}{ }^{b}+|\mathbb{D} u-\mathbb{D} l|^{p-1}+|\mathbb{D} u-\mathbb{D} l| \\
\leq & C(M) \sup _{Q_{\varrho}\left(z_{0}\right)}|\mathbb{D} \varphi|\left[\frac{\left|Q_{\varrho}^{b}\right|}{\left|Q_{\varrho}\right|}+\left(f-|\mathbb{D} u-\mathbb{D} l|^{p}\right)^{1 / p^{\prime}} \frac{\left|Q_{\varrho}^{b}\right|^{1 / p}}{\left|Q_{\varrho}\right|^{1 / p}}\right] \\
& +C(M) \sup _{Q_{\varrho}\left(z_{0}\right)}|\mathbb{D} \varphi|\left[\left(f|\mathbb{D} u-\mathbb{D} l|^{p}\right)^{1 / p} \frac{\left|Q_{\varrho}^{b}\right|^{1 / p^{\prime}}}{\left|Q_{\varrho}\right|^{1 / p^{\prime}}}\right] \\
\leq & C(M) \sup _{Q_{\varrho}\left(z_{0}\right)}|\mathbb{D} \varphi| \phi_{p}
\end{aligned}
$$

where the last inequality holds in view of (5.15) with $s=p$ and $s=p^{\prime}$. Combine estimates (5.14) and (5.17) to get

$$
\begin{aligned}
& f_{Q_{\varrho}\left(z_{0}\right)}\left[A\left(z_{0}, l\left(z_{0}\right), \mathbb{D} u\right)-\frac{\partial A}{\partial q}\left(z_{0}, l\left(z_{0}\right), \mathbb{D} l\right) \cdot(\mathbb{D} u-\mathbb{D} l)\right] \mathbb{D} \varphi \\
& \leq C(M)\left[\omega\left(M+1, \phi_{p}\right) \phi_{p}^{1 / p}+\phi_{p}\right] \sup _{Q_{\varrho}\left(z_{0}\right)}|\mathbb{D} \varphi| .
\end{aligned}
$$

It remains to estimate the first term in (5.7); use (5.2a) with $P \equiv \mathbb{D} u$ to get (2.1d)

$$
\left|A(z, u, \mathbb{D} u)-A\left(z_{0}, l\left(z_{0}\right), \mathbb{D} u\right)\right| \leq C(M) \varrho^{\beta}\left[1+|\mathbb{D} u-\mathbb{D} l|^{p}+\left|\frac{u-l}{\varrho}\right|^{p}\right]
$$

Inequality (5.19) used to estimate the first term of the right-hand-side of (5.6) gives

$$
\left|f_{Q_{\varrho}\left(z_{0}\right)}\left(A(z, u, \mathbb{D} u)-A\left(z_{0}, l\left(z_{0}\right), \mathbb{D} u\right)\right) \mathbb{D} \varphi\right| \leq \sup _{Q_{\varrho}\left(z_{0}\right)}|\mathbb{D} \varphi| C(M) \varrho^{\beta}\left[1+\psi_{p}+\phi_{p}\right]
$$

Inequalities (5.18), (5.20) used in (5.7) give thesis. 
Lemma 5.3. (Local inequalities) Let $u \in C\left(-T, 0 ; L^{2}(\Omega)\right) \cap L^{p}\left(-T, 0 ; W^{1, p}(\Omega)\right)$ be a weak solution to (1.1) with structure conditions $(2.1 c-2.2 a)$ and $p \geq 2$. Then the following inequalities hold for any $Q_{\varrho}\left(z_{0}\right)$ and constants being nondecreasing functions of their parameters

$$
\begin{aligned}
& \left|\int_{B_{\varrho}\left(x_{0}\right)}(u(t, x)-u(\tau, x)) \eta_{\varrho}(x) d x\right| \leq C_{(5.21)}\left(\left|(u)_{z_{0}}\right|+\left|(\mathbb{D} u)_{z_{0}}\right|\right) \varrho \\
& \quad \times f_{Q_{\varrho}\left(z_{0}\right)}\left(\varrho^{\beta}+\left|u-(u)_{z_{0}}\right|^{\beta}+\left|u-(u)_{z_{0}}\right|+\left|\mathbb{D} u-(\mathbb{D} u)_{z_{0}}\right|^{p-1}+\left|\mathbb{D} u-(\mathbb{D} u)_{z_{0}}\right|\right) \\
& \left|\int_{B_{\varrho}\left(x_{0}\right)}(u(t, x)-u(\tau, x)) \eta_{\varrho}(x) d x\right| \leq C_{(5.22)}\left(\left|(u)_{z_{0}}\right|+\left|(\mathbb{D} u)_{z_{0}}\right|\right) \varrho f_{Q_{\varrho}\left(z_{0}\right)} 1+|\mathbb{D} u|^{p-1}
\end{aligned}
$$

fort $\tau \in\left(t_{0}-\varrho^{2}, t_{0}\right)$, where $\eta_{\varrho}(x)$ denotes a standard mollifier in space, supported in $B_{\varrho}\left(x_{0}\right)$;

$$
\begin{array}{r}
\sup _{t \in\left(t_{0}-\left(\frac{\rho}{2}\right)^{2}, t_{0}\right)}|u-l|_{L^{2}\left(B_{\frac{\rho}{2}}\right)}^{2}+f_{Q_{\frac{\varrho}{2}\left(z_{0}\right)}}|\mathbb{D} u-\mathbb{D} l|^{2}+|\mathbb{D} u-\mathbb{D} l|^{p} \\
\leq C_{C a c c}(M)\left[f_{Q_{\varrho}\left(z_{0}\right)}\left|\frac{u-l}{\varrho}\right|^{2}+f_{Q_{\varrho}\left(z_{0}\right)}\left|\frac{u-l}{\varrho}\right|^{p}+\varrho^{2 \beta}\right] \\
f_{Q_{\frac{\varrho}{2}\left(z_{0}\right)}|\nabla u-\nabla l|^{2} \leq C_{C a c c}^{\prime}(M)}\left[f_{Q_{\varrho}\left(z_{0}\right)}\left|\frac{u-l}{\varrho}\right|^{2}+f_{Q_{\varrho}\left(z_{0}\right)}\left|\frac{u-l}{\varrho}\right|^{p}+\varrho^{2 \beta}\right]
\end{array}
$$

where $l$ is an affine function depending only on $x$ and satisfying $\left|l\left(z_{0}\right)\right|+|\nabla l| \leq M$ and $\beta \in(0,1)$ is given by $(2.1 d)$.

Proof. Fix arbitrary numbers $t, \tau$ and nonnegative $\varepsilon, \tilde{\varepsilon}$ satisfying

$$
t_{0}-\varrho^{2} \leq t<t+\tilde{\varepsilon}<\tau-\varepsilon<\tau \leq t_{0}
$$

and the continuous, piecewise affine cutoff function $\sigma(s) \in[0,1]$ defined by

$$
\begin{aligned}
& \sigma_{t, \tau, \varepsilon, \tilde{\varepsilon}}(s)= \begin{cases}1 & \text { on }(t+\tilde{\varepsilon}, \tau-\varepsilon), \\
0 & \text { on }(t, \tau)^{c},\end{cases} \\
& \sigma_{t, \tau, \varepsilon, \tilde{\varepsilon}}^{\prime}(s)= \begin{cases}1 / \tilde{\varepsilon} & \text { on }(t, t+\tilde{\varepsilon}), \\
-1 / \varepsilon & \text { on }(\tau-\varepsilon, \tau), \\
0 & \text { otherwise. }\end{cases}
\end{aligned}
$$


Let us first show (5.21). Test (1.1) with $\sigma_{t, \tau, \varepsilon, \varepsilon} \eta_{\varrho}$, obtaining

$$
\int_{B_{\varrho}\left(x_{0}\right)}(u(t, x)-u(\tau, x)) \eta_{\varrho}(x)=\int_{\tau}^{t} \int_{B_{\varrho}\left(x_{0}\right)} A(z, u, \mathbb{D} u) \mathbb{D} \eta_{\varrho}(x) d s
$$

by sending $\varepsilon \rightarrow 0$ (this holds pointwisely in time, because $u \in C\left(L^{2}\right)$ ). Estimate the r.h.s. of (5.26) using that $\left|\nabla \eta_{\varrho}(x)\right| \leq C \varrho^{-(d+1)}$

$$
\begin{aligned}
& \left|\int_{\tau}^{t} \int_{B_{\varrho}\left(x_{0}\right)} A(z, u, \mathbb{D} u) \mathbb{D} \eta_{\varrho}(x) d x d s\right| \\
& =\left|\int_{\tau}^{t} \int_{B_{\varrho}\left(x_{0}\right)}\left(A(z, u, \mathbb{D} u)-A\left(z_{0},(u)_{z_{0}},(\mathbb{D} u)_{z_{0}}\right)\right) \mathbb{D} \eta_{\varrho}(x) d x d s\right| \\
& \leq C \int_{Q_{\varrho}\left(z_{0}\right)}\left|A(z, u, \mathbb{D} u)-A\left(z_{0},(u)_{z_{0}},(\mathbb{D} u)_{z_{0}}\right)\right||\varrho|^{-(d+1)} \\
& \leq C_{\varrho} f_{Q_{\varrho}\left(z_{0}\right)}\left|A(z, u, \mathbb{D} u) \pm A\left(z, u,(\mathbb{D} u)_{z_{0}}\right)-A\left(z_{0},(u)_{z_{0}},(\mathbb{D} u)_{z_{0}}\right)\right| \\
& \leq C\left(\left|(u)_{z_{0}}\right|+\left|(\mathbb{D} u)_{z_{0}}\right|\right) \varrho \\
& \quad \times \quad f_{Q_{\varrho}\left(x_{0}\right)}\left(\varrho^{\beta}+\left|u-(u)_{z_{0}}\right|^{\beta}+\left|u-(u)_{z_{0}}\right|+\left|\mathbb{D} u-(\mathbb{D} u)_{z_{0}}\right|^{p-1}+\left|\mathbb{D} u-(\mathbb{D} u)_{z_{0}}\right|\right)
\end{aligned}
$$

where the last inequality comes from adding estimates (5.2b), (5.3) with $P \equiv \mathbb{D} u$ and $l(x) \equiv(u)_{z_{0}}+(\mathbb{D} u)_{z_{0}}\left(x-x_{0}\right)$. This ends the proof of (5.21). To get (5.22), when estimating (5.27), we use inequality (5.4) instead of (5.2b) and (5.3).

Let us now turn our attention to the energy estimate (5.23). To show it, choose a smooth cutoff function $\theta(x) \in[0,1]$ satisfying

$$
\begin{aligned}
& \theta(x)= \begin{cases}1 & \text { on } B_{\varrho / 2}\left(z_{0}\right), \\
0 & \text { on } B_{\varrho}^{c}\left(z_{0}\right),\end{cases} \\
& |\nabla \theta| \leq 4 / \varrho .
\end{aligned}
$$

Test (1.1) with $\varphi=(u-l) \theta^{2}(x) \sigma_{t_{0}-\varrho^{2}, \tau, \varepsilon, \frac{3}{4} \varrho^{2}}(s)$, suppressing for now parameters of cutoff function in time, thus writing $\sigma$. The evolutionary part yields 


$$
\begin{aligned}
& \int_{Q_{\varrho}\left(z_{0}\right)} u \varphi_{, s}=\int_{Q_{\varrho}\left(z_{0}\right)}(u-l) \varphi_{, s}=\int_{Q_{\varrho}\left(z_{0}\right)}(u-l) \theta^{2}\left(\sigma_{, s}(u-l)+\sigma(u-l)_{, s}\right) \\
& =\int_{Q_{\varrho}\left(z_{0}\right)}|u-l|^{2} \theta^{2} \sigma_{, s}+\frac{1}{2} \int_{Q_{\varrho}\left(z_{0}\right)}\left(|u-l|^{2} \theta^{2}\right)_{, s} \sigma=\frac{1}{2} \int_{Q_{\varrho}\left(z_{0}\right)}|u-l|^{2} \theta^{2} \sigma_{, s} \\
& \leq \int_{B_{\varrho} \times\left(t_{0}-\varrho^{2}, t_{0}-\frac{\varrho^{2}}{4}\right)}\left|\frac{u-l}{\varrho}\right|^{2} \theta^{2}-\frac{1}{\varepsilon} \int_{B_{\varrho} \times(\tau-\varepsilon, \tau)}|u-l|^{2} \theta^{2}
\end{aligned}
$$

the last inequality holds, because $\left|\sigma^{\prime}\right| \leq 2 \varrho^{-2}$ on $\left(t_{0}-\varrho^{2}, t_{0}-\varrho^{2} / 4\right)$ in view of (5.25). As $\int_{Q_{\varrho}\left(z_{0}\right)} A\left(z_{0}, l\left(z_{0}\right), \mathbb{D} l\right) \mathbb{D} \varphi=0$, for the main part holds

$$
\begin{aligned}
& \int_{Q_{\varrho}\left(z_{0}\right)} A(z, u, \mathbb{D} u) \mathbb{D} \varphi=\int_{Q_{\varrho}\left(z_{0}\right)}[A(z, u, \mathbb{D} u)-A(z, u, \mathbb{D} l)] \mathbb{D}(u-l) \theta^{2} \sigma \\
& +\int_{Q_{\varrho}\left(z_{0}\right)}[A(z, u, \mathbb{D} u)-A(z, u, \mathbb{D} l)] 2 \theta \nabla \theta:(u-l) \sigma \\
& +\int_{Q_{\varrho}\left(z_{0}\right)}\left[A(z, u, \mathbb{D} l)-A\left(z_{0}, l\left(z_{0}\right), \mathbb{D} l\right)\right] \mathbb{D} \varphi .
\end{aligned}
$$

For $p \geq 2$ holds

$$
|A-B|^{2} \int_{0}^{1}\left(1+|s(A-B)+B|^{2}\right)^{\frac{p-2}{2}} d s \geq c\left(|A-B|^{2}+|A-B|^{p}\right)
$$

hence, using the assumption (2.2a) one obtains

$$
\begin{aligned}
& {[A}(z, u, \mathbb{D} u)-A(z, u, \mathbb{D} l)] \mathbb{D}(u-l) \\
&= \int_{0}^{1} \frac{\partial A}{\partial q}(z, u, s(\mathbb{D} u-\mathbb{D} l)+\mathbb{D} l)(\mathbb{D} u-\mathbb{D} l) \cdot(\mathbb{D} u-\mathbb{D} l) d s \\
& \geq \int_{0}^{1} \lambda\left(1+|s(\mathbb{D} u-\mathbb{D} l)+\mathbb{D} l|^{2}\right)^{\frac{p-2}{2}}|\mathbb{D} u-\mathbb{D} l|^{2} d s \\
& \geq c\left(|\mathbb{D} u-\mathbb{D} l|^{p}+|\mathbb{D} u-\mathbb{D} l|^{2}\right)
\end{aligned}
$$

Inequalities (5.29), (5.30), (5.32) show that testing (1.1) with $\varphi=\theta^{2} \sigma(u-l)$ yields the following estimate

$$
\begin{aligned}
& \frac{1}{\varepsilon} \int_{B_{\varrho} \times(\tau-\varepsilon, \tau)}|u-l|^{2} \theta^{2}+\int_{Q_{\varrho}\left(z_{0}\right)}|\mathbb{D}(u-l)|^{2} \theta^{2} \sigma+\int_{Q_{\varrho}\left(z_{0}\right)}|\mathbb{D}(u-l)|^{p} \theta^{2} \sigma \\
& \quad \leq C \int_{Q_{\varrho}\left(z_{0}\right)}\left|\frac{u-l}{\varrho}\right|^{2}+C \int_{Q_{\varrho}\left(z_{0}\right)}|[A(z, u, \mathbb{D} u)-A(z, u, \mathbb{D} l)] 2 \theta \nabla \theta:(u-l) \sigma|
\end{aligned}
$$




$$
\begin{aligned}
& +C \int_{Q_{\varrho}\left(z_{0}\right)}\left|\left[A(z, u, \mathbb{D} l)-A\left(z_{0}, l\left(z_{0}\right), \mathbb{D} l\right)\right] \mathbb{D} \varphi\right| \\
\equiv & C \int_{Q_{\varrho}\left(z_{0}\right)}\left|\frac{u-l}{\varrho}\right|^{2}+I+I I
\end{aligned}
$$

Let us estimate $I$ by (5.3) with $P \equiv \mathbb{D} u$ getting for $\varrho \leq 1$

$$
\begin{aligned}
I & \leq C(M) \int_{Q_{\varrho}\left(z_{0}\right)}\left(|u-l|^{\beta}+|u-l|+|\mathbb{D} u-\mathbb{D} l|^{p-1}+|\mathbb{D} u-\mathbb{D} l|\right)\left|\frac{u-l}{\varrho}\right| \sigma \theta \\
& \leq \frac{1}{4} \int_{Q_{\varrho}\left(z_{0}\right)}|\mathbb{D} u-\mathbb{D} l|^{2} \theta^{2} \sigma+\frac{1}{2} \int_{Q_{\varrho}\left(z_{0}\right)}|\mathbb{D} u-\mathbb{D} l|^{p} \theta^{2} \sigma \\
& +C(M) \int_{Q_{\varrho}\left(z_{0}\right)}\left|\frac{u-l}{\varrho}\right|^{2}+\left|\frac{u-l}{\varrho}\right|^{p}+\frac{|u-l|^{\beta+1}}{\varrho} .
\end{aligned}
$$

Use (5.2b) to obtain

$$
\begin{aligned}
& \left|A\left(z_{0}, l\left(z_{0}\right), \mathbb{D} l\right)-A(z, u, \mathbb{D} l)\right||\mathbb{D} \varphi| \\
& \quad \leq C(M) \varrho^{\beta}\left[1+|u-l|^{\beta}\right]\left(\frac{|u-l|}{\varrho}+|\mathbb{D} u-\mathbb{D} l|\right) \theta \sigma
\end{aligned}
$$

with which we estimate $I I$

$$
\begin{aligned}
I I & \leq C(M) \int_{Q_{\varrho}\left(z_{0}\right)}\left[\varrho^{\beta}|\mathbb{D} u-\mathbb{D} l|+\varrho^{\beta} \frac{|u-l|}{\varrho}+\frac{|u-l|^{\beta+1}}{\varrho}+|u-l|^{\beta}|\mathbb{D} u-\mathbb{D} l|\right] \theta \sigma \\
& \leq \frac{1}{4} \int_{Q_{\varrho}\left(z_{0}\right)}|\mathbb{D} u-\mathbb{D} l|^{2} \theta^{2} \sigma+C(M) \int_{Q_{\varrho}\left(z_{0}\right)}\left[\varrho^{2 \beta}+|u-l|^{2 \beta}+\left|\frac{u-l}{\varrho}\right|^{2}+\frac{|u-l|^{\beta+1}}{\varrho}\right]
\end{aligned}
$$

Estimates for $I$ and II give together

$$
\begin{aligned}
& I+I I \leq \frac{1}{2} \int_{Q_{\varrho}\left(z_{0}\right)}\left[|\mathbb{D} u-\mathbb{D} l|^{p}+|\mathbb{D} u-\mathbb{D} l|^{2}\right] \theta^{2} \sigma \\
& +C(M) \int_{Q_{\varrho}\left(z_{0}\right)}\left[\varrho^{2 \beta}+|u-l|^{2 \beta}+\left|\frac{u-l}{\varrho}\right|^{2}+\left|\frac{u-l}{\varrho}\right|^{p}+\frac{|u-l|^{\beta+1}}{\varrho}\right]
\end{aligned}
$$

In view of $\beta<1, \varrho \leq 1$ one has

$$
\begin{aligned}
& \int_{Q_{\varrho}\left(z_{0}\right)}|u-l|^{2 \beta} \leq \varrho^{2 \beta} \int_{Q_{\varrho}\left(z_{0}\right)}\left|\frac{u-l}{\varrho}\right|^{2 \beta} \leq \varrho^{2 \beta}\left|Q_{\varrho}\right|+C \int_{Q_{\varrho}\left(z_{0}\right)}\left|\frac{u-l}{\varrho}\right|^{2}, \\
& \int_{Q_{\varrho}\left(z_{0}\right)} \frac{|u-l|^{\beta+1}}{\varrho} \leq \varrho^{\frac{2 \beta}{1-\beta}}\left|Q_{\varrho}\right|+\int_{Q_{\varrho}\left(z_{0}\right)}\left|\frac{u-l}{\varrho}\right|^{2} \leq \varrho^{2 \beta}\left|Q_{\varrho}\right|+\int_{Q_{\varrho}\left(z_{0}\right)}\left|\frac{u-l}{\varrho}\right|^{2}
\end{aligned}
$$


Consequently, (5.37) takes the form

$$
\begin{gathered}
I+I I \leq \frac{1}{2} \int_{Q_{\varrho}\left(z_{0}\right)}\left[|\mathbb{D} u-\mathbb{D} l|^{p}+|\mathbb{D} u-\mathbb{D} l|^{2}\right] \theta^{2} \sigma \\
+C(M)\left[\varrho^{2 \beta}\left|Q_{\varrho}\right|+\int_{Q_{\varrho}\left(z_{0}\right)}\left|\frac{u-l}{\varrho}\right|^{2}+\left|\frac{u-l}{\varrho}\right|^{p}\right] .
\end{gathered}
$$

hence (5.33) with (5.38) yields

$$
\begin{aligned}
& \frac{1}{\varepsilon} \int_{B_{\varrho / 2}\left(z_{0}\right) \times(\tau-\varepsilon, \tau)}|u-l|^{2}+\int_{t_{0}-\left(\frac{\varrho}{2}\right)^{2}}^{\tau-\varepsilon} \int_{B_{\varrho / 2}\left(z_{0}\right)}|\mathbb{D}(u-l)|^{p}+|\mathbb{D}(u-l)|^{2} \\
& \leq C(M)\left[\varrho^{2 \beta}\left|Q_{\varrho}\right|+\int_{Q_{\varrho}\left(z_{0}\right)}\left|\frac{u-l}{\varrho}\right|^{2}+\left|\frac{u-l}{\varrho}\right|^{p}\right] .
\end{aligned}
$$

First, use inequality (5.39) for $\tau=t_{0}$, neglecting the first term of the left-hand-side. This estimate is uniform in $\varepsilon$, so we obtain

$$
\begin{aligned}
& f_{Q_{\frac{\varrho}{2}}\left(z_{0}\right)}|\mathbb{D} u-\mathbb{D} l|^{2}+f_{Q_{\frac{\varrho}{2}}\left(z_{0}\right)}|\mathbb{D} u-\mathbb{D} l|^{p} \\
& \leq C(M)\left[f_{Q_{\varrho}\left(z_{0}\right)}\left|\frac{u-l}{\varrho}\right|^{2}+f_{Q_{\varrho}\left(z_{0}\right)}\left|\frac{u-l}{\varrho}\right|^{p}+\varrho^{2 \beta}\right]
\end{aligned}
$$

Next, drop second part of left-hand-side of (5.39) and consider any $\tau$ in interval of admissibility $\left(t_{0}-\frac{\rho^{2}}{4}, t_{0}\right]$; this gives rise to

$$
\sup _{t \in\left(t_{0}-\left(\frac{\varrho}{2}\right)^{2}, t_{0}\right)}|u-l|_{L^{2}\left(B_{\frac{\varrho}{2}}\right)}^{2} \leq C(M)\left[f_{Q_{\varrho}\left(z_{0}\right)}\left|\frac{u-l}{\varrho}\right|^{2}+f_{Q_{\varrho}\left(z_{0}\right)}\left|\frac{u-l}{\varrho}\right|^{p}+\varrho^{2 \beta}\right] .
$$

Combining (5.40) and (5.41) we have the first Caccioppoli estimate (5.23). It implies, in conjunction with the Korn's inequality (4.6a) used for $(u-l)(t)$, the 
following estimate

$$
\begin{aligned}
& f \underset{t_{0}-\frac{\varrho^{2}}{4}}{t_{B}} f_{\frac{\rho}{2}\left(x_{0}\right)}|\nabla u-\nabla l|^{2} \leq f_{t_{0}-\frac{\varrho^{2}}{4}}^{t_{0}} C_{K o r_{2}}\left[\underset{B_{\frac{\rho}{2}}\left(x_{0}\right)}{f}|\mathbb{D} u-\mathbb{D} l|^{2}+\underset{B_{\frac{\rho}{2}}\left(x_{0}\right)}{f}\left|\frac{u-l}{\varrho / 2}\right|^{2}\right] \\
& \leq C_{K o r_{2}} C_{C a c c}(M)\left[f_{Q_{\varrho}\left(z_{0}\right)}\left|\frac{u-l}{\varrho}\right|^{2}+f_{Q_{\varrho}\left(z_{0}\right)}\left|\frac{u-l}{\varrho}\right|^{p}+\varrho^{2 \beta}\right] \\
& +C_{K o r_{2}} 2^{n+4} f_{Q_{\varrho}\left(z_{0}\right)}\left|\frac{u-l}{\varrho}\right|^{2},
\end{aligned}
$$

which justifies (5.24) with $C_{C a c c}^{\prime} \equiv C_{K o r_{2}}\left(C_{C a c c}(M)+2^{n+4}\right)$.

Next, we restate the linearization lemma (Lemma 5.2) using local inequalities of Lemma 5.3 in a way useful for further computations. To proceed, introduce the following useful quantities

Definition 5.2. $E_{z_{0}, l}(\varrho)$ denotes the $L^{2}-L^{p}$ excess energy

$$
E_{z_{0}, l}(\varrho):=\psi_{2, z_{0}, l}(\varrho)+\psi_{p, z_{0}, l}(\varrho) \quad\left(=f_{Q_{z_{0}}(\varrho)}\left|\frac{u-l}{\varrho}\right|^{2}+\underset{Q_{z_{0}}(\varrho)}{f}\left|\frac{u-l}{\varrho}\right|^{p}\right)
$$

and $\tilde{E}_{z_{0}, l}(\varrho)$ denotes the perturbed $L^{2}-L^{p}$ excess energy

$$
\tilde{E}_{z_{0}, l}(\varrho):=E_{z_{0}, l}(\varrho)+\varrho^{2 \beta}
$$

where $\psi_{2, z_{0}, l}(\varrho), \psi_{p, z_{0}, l}(\varrho)$ are given as in Definition 5.1.

Definition 5.3. Introduce normalization factor $\gamma_{l, \delta}(\varrho)$

$$
\gamma_{l, \delta}(\varrho):=\sqrt{E_{z_{0}, l}(\varrho)+(\delta / 2)^{-2} \varrho^{2 \beta}}
$$

Corollary 5.4. Let $u \in C\left(-T, 0 ; L^{2}(\Omega)\right) \cap L^{p}\left(-T, 0 ; W^{1, p}(\Omega)\right)$ be a weak solution to (1.1) with structure conditions (2.1a-2.2a), (2.2c-2.2d). Take $p \geq 2$ and fix $M$. There exists such constant $C_{5.4}(M)$ that for any affine function $l$ depending only on $x$ and satisfying $\left|l\left(x_{0}\right)\right|+|\nabla l| \leq M$ and any $\delta \in(0,1)$ for

$$
v:=\frac{u-l}{C_{5.4}(M) \gamma_{l, \delta}(\varrho)}
$$


the following inequalities hold

$$
\begin{aligned}
& \left|\int_{Q_{\varrho / 2}\left(z_{0}\right)} v \varphi_{, t}-\frac{\partial A}{\partial q}\left(z_{0}, l\left(z_{0}\right), \mathbb{D} l\right) \mathbb{D} v \mathbb{D} \varphi\right| \\
& \leq\left[\omega\left(M+1, \tilde{E}_{z_{0}, l}(\varrho)\right)+\tilde{E}_{z_{0}, l}^{\frac{1}{2}}(\varrho)+\delta / 2\right] \sup _{Q_{\varrho / 2}\left(z_{0}\right)}|\mathbb{D} \varphi|
\end{aligned}
$$

and

$$
f_{Q_{\varrho / 2}\left(z_{0}\right)}\left|\frac{v}{\varrho / 2}\right|^{2}+|\nabla v|^{2}+\left(C_{5.4}(M) \gamma_{l, \delta}(\varrho)\right)^{p-2}\left[\left|\frac{v}{\varrho / 2}\right|^{p}+|\nabla v|^{p}\right] \leq 1,
$$

where $Q_{\varrho}\left(z_{0}\right) \subset Q$ is an arbitrary local cylinder with $\varrho \leq 1$.

Proof. We suppress parameters of the excess energies writing $E, \tilde{E}$ for $E_{z_{0}, l}, \tilde{E}_{z_{0}, l}$ and similarily for moments $\psi, \phi$. Take

$$
C_{5.4}(M) \equiv 2^{n+2+p}\left(2 \max _{i=2, p}\left(C_{K o r_{i}}\right)\right)^{1 / 2} C_{\text {lin }}(M)\left(1+C_{C a c c}^{1+\frac{1}{p}}(M)\right)
$$

with this choice of $C_{5.4}(M)$, (5.50) yields (5.47).

Assumptions of Lemmas 5.2, 5.3 are fulfilled. Linearization inequality (5.5) with the Caccioppoli estimate (5.23) give

$$
\begin{aligned}
& \left|\int_{Q_{\varrho / 2}\left(z_{0}\right)}(u-l) \varphi_{, t}-\frac{\partial A}{\partial q}\left(z_{0}, l\left(z_{0}\right), \mathbb{D} l\right)(\mathbb{D} u-\mathbb{D} l) \mathbb{D} \varphi\right| \\
& \leq C_{l i n}(M)\left[\omega\left(M+1, \phi_{p}\left(\frac{\varrho}{2}\right)\right) \phi_{2}^{\frac{1}{2}}\left(\frac{\varrho}{2}\right)+\phi_{p}\left(\frac{\varrho}{2}\right)+\psi_{p}\left(\frac{\varrho}{2}\right)+\left(\frac{\varrho}{2}\right)^{\beta}\right] \sup _{Q_{\varrho / 2}\left(z_{0}\right)}|\mathbb{D} \varphi| \\
& \leq C_{l i n}(M) C_{C a c c}(M) \\
& \quad \times\left[\omega\left(M+1, C_{C a c c}(M) \tilde{E}(\varrho)\right) \tilde{E}^{\frac{1}{2}}(\varrho)+\tilde{E}(\varrho)+2^{n+2+p} E(\varrho)+\varrho^{\beta}\right]_{\sup _{\varrho / 2}\left(z_{0}\right)}|\mathbb{D} \varphi| \\
& \leq 2^{n+2+p} C_{l i n}(M) C_{C a c c}^{1+\frac{1}{p}}(M) \gamma_{l, \delta}(\varrho)\left[\omega(M+1, \tilde{E}(\varrho))+\tilde{E}^{\frac{1}{2}}(\varrho)+\frac{\delta}{2}\right]_{\sup _{\varrho / 2}\left(z_{0}\right)}|\mathbb{D} \varphi|
\end{aligned}
$$

The last inequality holds by concavity of $\omega^{p}$ with respect to its second variable giving for $c>1: \omega(M+1, c \alpha) \leq c^{\frac{1}{p}} \omega(M+1, \alpha)$, and by definition (5.45) of $\gamma_{l, \delta}(\varrho)$. From (5.50) we have (5.47). Let us now justify inequality (5.48). Using Korn's inequality (4.6a) from Lemma 4.3 compute 


$$
\begin{aligned}
& f_{Q_{\varrho / 2}\left(z_{0}\right)}\left|\frac{v}{\varrho / 2}\right|^{2}+|\nabla v|^{2}+\left(C_{5.4}(M) \gamma_{l, \delta}(\varrho)\right)^{p-2}\left[\left|\frac{v}{\varrho / 2}\right|^{p}+|\nabla v|^{p}\right] \\
& \leq 2 \max _{i=2, p}\left(C_{K o r_{i}}\right) f_{Q_{\varrho / 2}\left(z_{0}\right)}\left|\frac{v}{\varrho / 2}\right|^{2}+|\mathbb{D} v|^{2}+\left(C_{5.4}(M) \gamma_{l, \delta}(\varrho)\right)^{p-2}\left[\left|\frac{v}{\varrho / 2}\right|^{p}+|\mathbb{D} v|^{p}\right] \\
& \leq\left(2^{n+2+p} C_{l i n}(M)\left(1+C_{C a c c}^{1+\frac{1}{p}}(M)\right) \gamma_{l, \delta}(\varrho)\right)^{-2} \\
& \times \int_{Q_{\varrho / 2}\left(z_{0}\right)}|\mathbb{D}(u-l)|^{2}+\left|\frac{u-l}{\varrho / 2}\right|^{2}+|\mathbb{D}(u-l)|^{p}+\left|\frac{u-l}{\varrho / 2}\right|^{p} \\
& \leq\left(2^{n+2+p} C_{l i n}(M)\left(1+C_{C a c c}^{1+\frac{1}{p}}(M)\right)\right)^{-2} \\
& \times \tilde{E}_{z 0, l}^{-1}(\varrho) f_{Q_{\varrho / 2}\left(z_{0}\right)}|\mathbb{D}(u-l)|^{2}+\left|\frac{u-l}{\varrho / 2}\right|^{2}+|\mathbb{D}(u-l)|^{p}+\left|\frac{u-l}{\varrho / 2}\right|^{p} \\
& \leq\left(2^{n+2+p} C_{l i n}(M)\left(1+C_{C a c c}^{1+\frac{1}{p}}(M)\right)\right)^{-2}\left(2^{n+2+p}+C_{C a c c}(M)\right)
\end{aligned}
$$

The last three inequalities come, respectively, from: the definition (5.46) of $v$ and choice (5.49) of constant $C_{5.4}(M)$; the Definition 5.3 of $\gamma_{l, \delta}(\varrho)$ and the fact that $\delta \leq 1$; the Caccioppoli inequality (5.23) and the Definition 5.2 of perturbed excess energy $\tilde{E}$. As $C_{\text {lin }}, C_{\text {Cacc }}$ are bigger than 1, (5.51) implies (5.48).

\section{Partial regularity}

First we merge the local inequality of Corollary 5.4 and the caloric approximation into a building block of a further partial regularity result. Recall from Lemma 4.2 that $l_{z_{0}, \varrho}^{(s)}(x)$ is the affine function, depending only on space variable, which minimizes $f_{Q_{\varrho}\left(z_{0}\right)}|u-l|^{s} ; l_{\varrho}^{(s)}(x)$ denotes this function, when dependence on $z_{0}$ is irrelevant.

Lemma 6.1. Let $p \geq 2$ and $u \in C\left(-T, 0 ; L^{2}(\Omega)\right) \cap L^{p}\left(-T, 0 ; W^{1, p}(\Omega)\right)$ be a weak solution to (1.1) under structure conditions $(2.1 a-2.2 d)$. Fix constants $M>$ $0, \alpha \in(0,1)$. Then there exist $\sigma \in(0,1 / 4), \delta \in(0,1)$, such that for any $\varrho<1$, $z_{0} \in Q$ (such that $Q_{\varrho}\left(z_{0}\right) \subset Q$ ), we have the following implication.

If

$$
\left|l_{z_{0}, \varrho}^{(2)}\left(x_{0}\right)\right|+\left|\nabla l_{z_{0}, \varrho}^{(2)}\right| \leq M \text {. }
$$

and

$$
\omega\left(M+1, \tilde{E}_{z_{0}, l_{z_{0}, \varrho}^{(2)}}(\varrho)\right)+\tilde{E}_{z_{0}, l_{z_{0}, \varrho}^{(2)}}^{\frac{1}{2}}(\varrho) \leq \frac{\delta}{2 C_{5.4}(M)},
$$


then

$$
\tilde{E}_{z_{0}, l_{z_{0}, \sigma \varrho}^{(2)}}(\sigma \varrho) \leq \sigma^{2 \alpha}\left[E_{z_{0}, l_{z_{0}, \varrho}^{(2)}}(\varrho)+\delta^{-2} \varrho^{2 \beta}\right]+(\sigma \varrho)^{2 \beta}
$$

Proof. We need certain care to avoid a logical loop. Therefore let us first explicitly define constants:

$$
\begin{aligned}
\tilde{C}_{(4.9)}(\lambda, \Lambda, s) & :=C_{(4.9)}(\lambda, \Lambda, 1, d, s, \infty), \\
\tilde{C}_{(4.11)}(\lambda, \Lambda, s) & :=C_{(4.11)}(\lambda, \Lambda, 0, d, s, s), \\
C_{(6.4)} & :=C_{5.4}^{2}(M) 2^{5 p-3} \max _{s \in\{2 ; p\}}\left(1+C_{(4.9)}(\lambda, \Lambda, s)+C_{(4.11)}(\lambda, \Lambda, s)\right) .
\end{aligned}
$$

We have already fixed in the statement of lemma $M>0, \alpha \in(0,1)$. Now let us fix certain parameters:

$$
\begin{aligned}
\sigma & \left.<1 / 4 \text { so that } C_{(6.4)} 2^{7} \sigma^{2} \leq \sigma^{2 \alpha} \quad \text { (which is possible as } \alpha \in(0,1)\right) \\
\varepsilon & \equiv(4 \sigma)^{p+d+2} 16 \sigma^{2} \\
\theta & \equiv 4 \sigma \\
\Lambda & =C_{(2.2 \mathrm{c})}(M)
\end{aligned}
$$

Observe that by assumptions (2.2a), (2.2b) holds

$$
\frac{\partial A}{\partial q}\left(z_{0}, l_{\varrho}^{(2)}\left(z_{0}\right), \mathbb{D} l_{\varrho}^{(2)}\right) \in S\left(\lambda,\left|\frac{\partial A}{\partial q}\left(z_{0}, l_{\varrho}^{(2)}\left(z_{0}\right), \mathbb{D} l_{\varrho}^{(2)}\right)\right|\right) \subset S(\lambda, \Lambda)
$$

i.e. the constant coefficients matrix, resulting from linearization around $z_{0}$, belongs to the set of elliptic bilinear, symmetrizing forms as defined in Definition 4.1. The imbedding results from (2.2c) with (6.1); $\lambda$ is given by (2.2a) and $\Lambda$-by (6.5). Consequently, let us fix via Lemma 4.5

$$
\delta_{\varepsilon} \equiv \delta(\varepsilon, \lambda, \Lambda)
$$

Take

$$
\gamma \equiv C_{5.4}(M) \gamma_{l_{\varrho}^{(2)}, \delta_{\varepsilon}}(\varrho)
$$

Observe that assumptions of Corollary 5.4 are fulfilled; this and assumption (6.2) give for $v$ defined as in (5.46), inequalities

$$
\begin{gathered}
\left|\int_{Q_{\varrho / 2}\left(z_{0}\right)} v \varphi_{, t}-\frac{\partial A}{\partial q}\left(z_{0}, l_{\varrho}^{(2)}\left(z_{0}\right), \mathbb{D} l_{\varrho}^{(2)}\right) \mathbb{D} v \mathbb{D} \varphi\right| \leq \delta_{\varepsilon} \sup _{Q_{\varrho / 2}\left(z_{0}\right)}|\mathbb{D} \varphi| \\
f_{Q_{\varrho / 2}\left(z_{0}\right)}\left|\frac{v}{\varrho / 2}\right|^{2}+\gamma^{p-2}\left|\frac{v}{\varrho / 2}\right|^{p}+|\nabla v|^{2}+\gamma^{p-2}|\nabla v|^{p} \leq 1
\end{gathered}
$$


By (6.8) and (6.2) one has also

$$
0 \leq \gamma=C_{5.4}(M) \sqrt{E_{z_{0}, l_{\varrho}^{(2)}}(\varrho)+\left(\delta_{\varepsilon} / 2\right)^{-2} \varrho^{2 \beta}} \leq \frac{C_{5.4}(M)}{\delta} \sqrt{\tilde{E}_{z_{0}, l_{\varrho}^{(2)}}(\varrho)} \leq 1 .
$$

Obervation (6.6) with inequalities (6.9), (6.10), (6.11) imply that $v$ belongs to the set $H(\varrho / 2 ; \delta, \Lambda, \gamma)$ of approximatively weakly symmetrical caloric functions (compare Definition 4.2). Consequently in view of the symmetric caloric approximation lemma, i.e. Lemma 4.5, there exists a caloric function $h$ that locally approximates $v$; more precisely

$$
\exists h \in H(\varrho / 4 ; \Lambda, \gamma) \quad \text { such that } f_{Q_{\varrho / 4}\left(z_{0}\right)}\left|\frac{h-v}{\varrho / 4}\right|^{2}+\gamma^{p-2}\left|\frac{h-v}{\varrho / 4}\right|^{p} \leq \varepsilon
$$

Having such approximation of $v$ by $h$, we are ready to show (6.3); to this end, estimate $\psi_{s, z_{0}, l_{\sigma \varrho}^{(2)}}(\sigma \varrho)$, (which for $s$ being $2, p$ constitute by definition $\tilde{E}_{z_{0}, l_{\sigma \varrho}^{(2)}}(\sigma \varrho)$ ) as follows

$$
\begin{aligned}
& \left(\frac{\theta \varrho}{4}\right)^{-s} f_{Q \frac{\varrho \theta}{4}\left(z_{0}\right)}\left|u-l_{\frac{\theta \varrho}{4}}^{(2)}\right|^{s} \leq 2^{s-1}\left(\frac{\theta \varrho}{4}\right)^{-s}\left[\underset{Q_{\frac{\varrho \theta}{4}\left(z_{0}\right)}}{f}\left|u-l_{\frac{\theta \varrho}{4}}^{(s)}\right|^{s}+\left|l_{\frac{\theta \varrho}{4}}^{(2)}-l_{\frac{\theta \varrho}{4}}^{(s)}\right|^{s}\right] \\
& \leq 2^{s} C_{(4.5)}(n, s)\left(\frac{\theta \varrho}{4}\right)^{-s} f_{Q_{\frac{\rho \theta}{4}}\left(z_{0}\right)}\left|u-l_{\frac{\theta \varrho}{4}}^{(s)}\right|^{s} \\
& \leq 2^{s} C_{(4.5)}(n, s)\left(\frac{\theta \varrho}{4}\right)^{-s} f_{Q_{\frac{\varrho \theta}{4}}\left(z_{0}\right)}\left|u-l_{\varrho}^{(2)}-\gamma\left[(h)_{z_{0}, \frac{\theta \varrho}{4}}-(\nabla h)_{z_{0}, \frac{\varrho \theta}{4}}\left(x-x_{0}\right)\right]\right|^{s} \\
& =2^{s} C_{(4.5)}(n, s) \gamma^{s}\left(\frac{\theta \varrho}{4}\right)^{-s} f_{Q_{\frac{\varrho \theta}{4}}\left(z_{0}\right)}\left|v-(h)_{z_{0}, \frac{\theta \varrho}{4}}-(\nabla h)_{z_{0}, \frac{\rho \theta}{4}}\left(x-x_{0}\right)\right|^{s} \\
& \leq 2^{2 s-1} C_{(4.5)}(n, s) \theta^{-(s+n+2)} \gamma^{s} \int_{Q_{\varrho / 4}\left(z_{0}\right)}\left|\frac{h-v}{\varrho / 4}\right|^{s} \\
& +2^{2 s-1} C_{(4.5)}(n, s)\left(\frac{\theta \varrho}{4}\right)^{-s} \gamma^{s} \int_{Q_{\frac{\varrho \theta}{4}}\left(z_{0}\right)}\left|h-(h)_{z_{0}, \frac{\theta \varrho}{4}}-(\nabla h)_{z_{0}, \frac{\varrho \theta}{4}}\left(x-x_{0}\right)\right|^{s},
\end{aligned}
$$

where the second inequality holds in view of (4.5) of Lemma 4.2, the third one by minimization property of $l^{(s)}$ and the equality is given by definition (5.46) of $v$. To proceed further denote the mean integral over space (emphasizing its time dependence) by 


$$
(g)(t)_{x_{0}, \varrho} \equiv f_{B_{\varrho}\left(x_{0}\right)} g(x, t) d x
$$

and estimate the second integral in the r.h.s. of (6.13) as follows

$$
\begin{aligned}
& f_{Q_{\frac{\rho \theta}{4}}\left(z_{0}\right)}\left|h-(h)_{z_{0}, \frac{\theta \varrho}{4}}-(\nabla h)_{z_{0}, \frac{\rho \theta}{4}}\left(x-x_{0}\right)\right|^{s} \\
& \leq 2^{s-1} f_{Q_{\frac{\rho \theta}{4}}\left(z_{0}\right)}\left|h(x, t)-(h)_{x_{0}, \frac{\theta \varrho}{4}}(t)-(\nabla h)_{z_{0}, \frac{\rho \theta}{4}}\left(x-x_{0}\right)\right|^{s} d x d t \\
& +2^{s-1} f_{Q_{\frac{\rho \theta}{4}}\left(z_{0}\right)}\left|(h)_{x_{0}, \frac{\rho \theta}{4}}(t)-(h)_{z_{0}, \frac{\rho \theta}{4}}\right|^{s} d x d t
\end{aligned}
$$

Observe that we cannot take $(h)_{x_{0}, \frac{\rho \theta}{4}}(t)$ instead of $(h)_{z_{0}, \frac{\rho \theta}{4}}$ directly in the second inequality of (6.13), as only time-independent affine functions are admissible there.

Consider the right-hand-side of (6.14). One has

$$
\left(h-(h)_{x_{0}, \frac{\theta \varrho}{4}}(t)-(\nabla h)_{z_{0}, \frac{\rho \theta}{4}}\left(x-x_{0}\right)\right)_{x_{0}, \frac{\theta \varrho}{4}}=0
$$

for every $t$, so Poincaré inequality in space followed by integration over time gives

$$
\begin{aligned}
& \int_{Q_{\frac{\varrho \theta}{4}}\left(z_{0}\right)}\left|h-(h)_{x_{0}, \frac{\theta \varrho}{4}}(t)-(\nabla h)_{z_{0}, \frac{\varrho \theta}{4}}\left(x-x_{0}\right)\right|^{s} \leq \frac{(\theta \varrho)^{s}}{4^{s}} \underset{Q_{\frac{\varrho \theta}{4}}\left(z_{0}\right)}{f}\left|\nabla h-(\nabla h)_{z_{0}, \frac{\varrho \theta}{4}}\right|^{s} \\
& \leq \tilde{C}_{(4.11)}(\lambda, \Lambda, s) \theta^{s}\left(\frac{\theta \varrho}{4}\right)^{s} f_{Q_{\frac{\varrho}{4}}\left(z_{0}\right)}|\nabla h|^{s} \leq \tilde{C}_{(4.11)}(\lambda, \Lambda, s) \theta^{s}\left(\frac{\theta \varrho}{4}\right)^{s} \gamma^{2-s}
\end{aligned}
$$

the second inequality results from estimate (4.11) of Lemma 4.4 used with $m=$ $0, q=p=s$ for $\frac{\partial h}{\partial x_{i}}$; the last inequality is valid as $h$ is a symmetrical caloric function. Simultaneously we have thanks to a smoothness of $h$ and the mean-value property

$$
\begin{aligned}
& f_{Q_{\frac{\varrho \theta}{4}}\left(z_{0}\right)}\left|(h)_{x_{0}, \frac{\rho \theta}{4}}(t)-(h)_{z_{0}, \frac{\rho \theta}{4}}\right|^{s} \\
& =\int_{t_{0}-\left(\frac{\rho \theta}{4}\right)^{2}}^{t_{0}}\left|f_{t_{0}-\left(\frac{\rho \theta}{4}\right)^{2}}^{t_{0}}(h)_{x_{0}, \frac{\rho \theta}{4}}(t)-(h)_{x_{0}, \frac{\rho \theta}{4}}(r) d r\right|^{s} d t
\end{aligned}
$$




$$
\begin{aligned}
& \leq \int_{t_{0}-\left(\frac{\rho \theta}{4}\right)^{2}}^{t_{0}} f_{t_{0}-\left(\frac{\rho \theta}{4}\right)^{2}}^{t_{0}}\left|\sup _{\tau \in\left(t_{0}-\left(\frac{\varrho \theta}{4}\right)^{2}, t_{0}\right)}\left(\frac{\partial h}{\partial \tau}\right)_{x_{0}, \frac{\rho \theta}{4}}(\tau)\right||t-r| d r \mid d t \\
& \leq\left(\frac{\varrho \theta}{4}\right)^{2 s}\left|\sup _{\tau \in\left(t_{0}-\left(\frac{\varrho \theta}{4}\right)^{2}, t_{0}\right)}^{s}\left(\frac{\partial h}{\partial \tau}\right)_{x_{0}, \frac{\rho \theta}{4}}(\tau)\right|
\end{aligned}
$$

Using the inequality (4.9) of Lemma 4.4 with $m=1, q=\infty, p=s$ to estimate the r.h.s. of (6.16) one arrives at

$$
\begin{aligned}
\int_{Q_{\frac{\varrho \theta}{4}}\left(z_{0}\right)}\left|(h)_{x_{0}, \frac{\rho \theta}{4}}(t)-(h)_{z_{0}, \frac{\rho \theta}{4}}\right|^{s} & \leq \tilde{C}_{(4.9)}(\lambda, \Lambda, s)\left(\frac{\varrho \theta}{4}\right)^{2 s}\left(\frac{\varrho}{4}\right)^{-s} f_{Q_{\frac{\varrho}{4}}\left(z_{0}\right)}\left|\frac{h}{\varrho / 4}\right|^{s} \\
& \leq \tilde{C}_{(4.9)}(\lambda, \Lambda, s) \theta^{s}\left(\frac{\varrho \theta}{4}\right)^{s} \gamma^{2-s}
\end{aligned}
$$

where the second inequality results from $h$ being a symmetrical caloric function. Combine (6.15) and (6.17) to estimate the right-hand-side of (6.14)

$$
\begin{aligned}
& f_{Q_{\frac{\varrho \theta}{4}}\left(z_{0}\right)}\left|h-(h)_{z_{0}, \frac{\theta \varrho}{4}}-(\nabla h)_{z_{0}, \frac{\rho \theta}{4}}\left(x-x_{0}\right)\right|^{s} \\
& \quad \leq 2^{s-1}\left(\tilde{C}_{(4.9)}(\lambda, \Lambda, s)+\tilde{C}_{(4.11)}(\lambda, \Lambda, s)\right) \theta^{s}\left(\frac{\varrho \theta}{4}\right)^{s} \gamma^{2-s}
\end{aligned}
$$

This and (6.12) we use in (6.13) to get

$$
\begin{aligned}
& \left(\frac{\theta \varrho}{4}\right)^{-s} \underset{Q_{\frac{\varrho \theta}{4}}\left(z_{0}\right)}{f}\left|u-l_{\frac{\theta \varrho}{4}}^{(2)}\right|^{s} \leq 2^{2 s-1} C_{(4.5)}(n, s) \gamma^{2}\left[\theta^{-(s+n+2)} \varepsilon+\theta^{s} 2^{s-1}\right. \\
& \left.\quad \times\left(\tilde{C}_{(4.9)}(\lambda, \Lambda, s) \times+\tilde{C}_{(4.11)}(\lambda, \Lambda, s)\right)\right]
\end{aligned}
$$

Estimate (6.19) with $s=2$ and $s=p$ gives, in view of $\theta \leq 1$, Definition 5.2 of $\tilde{E}$ and choice (6.8) of $\gamma$

$$
\begin{aligned}
& E_{z 0, l_{\frac{\theta \varrho}{4}}^{(2)}}\left(\frac{\theta \varrho}{4}\right) \leq C_{5.4}^{2}(M)\left[E_{z_{0}, l_{\varrho}^{(2)}}(\varrho)+\left(\delta_{\varepsilon} / 2\right)^{-2} \varrho^{2 \beta}\right] \\
& \quad \times C_{(6.20)}(\lambda, \Lambda, p)\left[\theta^{-(p+n+2)} \varepsilon+\theta^{2}\right]
\end{aligned}
$$

where (robustly)

$$
C_{(6.20)} \equiv 2^{5 p-3} \max _{s \in\{2 ; p\}} C_{(4.5)}(n, s)\left(\tilde{C}_{(4.9)}(\lambda, \Lambda, s)+\tilde{C}_{(4.11)}(\lambda, \Lambda, s)\right)
$$


recall that we have taken $\sigma=\theta / 4$; this with the definition of $C_{(6.4)}$ gives from (6.20)

$$
\begin{aligned}
& \tilde{E}_{z_{0}, l_{\sigma \varrho}^{(2)}}(\sigma \varrho) \leq C_{(6.4)}\left[\frac{\varepsilon}{(4 \sigma)^{p+n+2}}+16 \sigma^{2}\right]\left[E_{z_{0}, l_{\varrho}^{(2)}}(\varrho)+\left(\delta_{\varepsilon} / 2\right)^{-2} \varrho^{2 \beta}\right]+(\sigma \varrho)^{2 \beta} \\
& \leq C_{(6.4)} 32 \sigma^{2} 4\left[E_{z_{0}, l_{\varrho}(2)}(\varrho)+\delta_{\varepsilon}^{-2} \varrho^{2 \beta}\right]+(\sigma \varrho)^{2 \beta}
\end{aligned}
$$

where the second inequality is given by choice of $\varepsilon$, see (6.5); in the same inequality we have chosen $\sigma$ so that $C_{(6.4)} 2^{7} \sigma^{2} \leq \sigma^{2 \alpha}$, which gives thesis.

Let us now state inequalities used for the singular set description in the following iteration of Lemma 6.1 performed in Lemma 6.3.

Lemma 6.2. Under the assumptions of Lemma 6.1, the following inequalities hold

$$
\begin{aligned}
& \varrho^{-p} \int_{Q_{\varrho}\left(z_{0}\right)}\left|u-l_{\varrho}^{(2)}\right|^{p} \\
& \leq C\left(\left|(u)_{z_{0}}\right|+\left|(\nabla u)_{z_{0}}\right|\right)\left[\varrho^{p \beta}+\max _{l=1, \beta}\left(f_{Q_{\varrho}\left(z_{0}\right)}\left|\nabla u-(\nabla u)_{z_{0}}\right|^{p}\right)^{l}\right] \\
& +C\left(\left|(u)_{z_{0}}\right|+\left|(\nabla u)_{z_{0}}\right|\right)\left[\max _{r=1, \beta(p-1), p-1}\left(f_{Q_{\varrho}\left(z_{0}\right)}\left|\mathbb{D} u-(\mathbb{D} u)_{z_{0}}\right|^{p}\right)^{r}\right]
\end{aligned}
$$$$
\varrho^{-p} f_{Q_{\varrho}\left(z_{0}\right)}\left|u-(u)_{z_{0}}-(\nabla u)_{z_{0}}\left(x-x_{0}\right)\right|^{p}
$$$$
\begin{aligned}
& \leq C\left(\left|(u)_{z_{0}}\right|+\left|(\nabla u)_{z_{0}}\right|\right)\left[\varrho^{p \beta}+\max _{l=1, \beta}\left(f_{Q_{\varrho}\left(z_{0}\right)}\left|\nabla u-(\nabla u)_{z_{0}}\right|^{p}\right)^{l}\right] \\
& +C\left(\left|(u)_{z_{0}}\right|+\left|(\nabla u)_{z_{0}}\right|\right) \max _{r=1, \beta(p-1), p-1}\left[\left(f_{Q_{\varrho}\left(z_{0}\right)}\left|\mathbb{D} u-(\mathbb{D} u)_{z_{0}}\right|^{p}\right)^{r}\right],
\end{aligned}
$$

$$
\begin{aligned}
& \varrho^{-p} \int_{Q_{\varrho}\left(z_{0}\right)}\left|u-(u)_{z_{0}}\right|^{p} \leq C\left(\left|(u)_{z_{0}}\right|+\left|(\nabla u)_{z_{0}}\right|\right) \\
& \quad \times\left(1+\left(f_{Q_{\varrho}\left(z_{0}\right)}\left|\mathbb{D} u-(\mathbb{D} u)_{z_{0}}\right|^{p}\right)^{p-1}+f_{Q_{\varrho}\left(z_{0}\right)}\left|\nabla u-(\nabla u)_{z_{0}}\right|^{p}\right) .
\end{aligned}
$$

Proof. Recall that $\eta_{\varrho}(x)$ denotes a standard space-mollifier, supported in $B_{\varrho}\left(x_{0}\right)$. Define 


$$
(u)_{\eta, x_{0}}(t) \equiv \int_{B_{\varrho}\left(z_{0}\right)} u(x, t) \eta_{\varrho}(x) d x, \quad(u)_{\eta, z_{0}} \equiv f_{t_{0}-\varrho^{2}}^{t_{0}}(u)_{\eta, x_{0}}(t)
$$

Let us perform estimates, using first (4.5), then the approximative minimization property of standard mean value with respect to $L^{p}$ norms

$$
\begin{aligned}
& f_{Q_{\varrho}\left(z_{0}\right)}\left|u-(u)_{z_{0}}-(\nabla u)_{z_{0}}\left(x-x_{0}\right)\right|^{p} \leq C \underset{Q_{\varrho}\left(z_{0}\right)}{f}\left|u-(u)_{\eta, z_{0}}-(\nabla u)_{z_{0}}\left(x-x_{0}\right)\right|^{p} \\
& \quad \leq C f_{Q_{\varrho}\left(z_{0}\right)}\left|u-(u)_{\eta, x_{0}}(t)-(\nabla u)_{z_{0}}\left(x-x_{0}\right)\right|^{p}+f_{Q_{\varrho}\left(z_{0}\right)}\left|(u)_{\eta, x_{0}}(t)-(u)_{\eta, z_{0}}\right|^{p} d x d t
\end{aligned}
$$

The first integral in the right-hand-side of (6.27) is majorized in view of Poincaré inequality in space (for generalized integral means) by

$$
C \varrho^{p} \int_{Q_{\varrho}\left(z_{0}\right)}\left|\nabla u-(\nabla u)_{z_{0}}\right|^{p}
$$

whereas for the second one, in view of the inequality (5.21) of Lemma 5.3 holds

$$
\begin{aligned}
& f_{Q_{\varrho}\left(z_{0}\right)}\left|(u)_{\eta, x_{0}}(t)-(u)_{\eta, z_{0}}\right|^{p}=\int_{t_{0}-\varrho^{2}}^{t_{0}}\left|\int_{t_{0}-\varrho^{2}}^{t_{0}}(u)_{\eta, x_{0}}(t)-(u)_{\eta, x_{0}}(r) d r\right|^{p} d t \\
& \leq f f_{t_{0}-\varrho^{2}}^{t_{0}} f_{t_{0}-\varrho^{2}}^{t_{0}}\left|\int_{B_{\varrho}\left(x_{0}\right)}(u(t, x)-u(r, x)) \eta_{\varrho}(x)\right|^{p} d r d t \leq C_{(5.21)}^{p}\left(\left|(u)_{z_{0}}\right|+\left|(\mathbb{D} u)_{z_{0}}\right|\right) \varrho^{p} \\
& \times\left|\int_{Q_{\varrho}\left(z_{0}\right)} \varrho^{\beta}+\right| u-\left.(u)_{z_{0}}\right|^{\beta}+\left|u-(u)_{z_{0}}\right|+\left|\mathbb{D} u-(\mathbb{D} u)_{z_{0}}\right|^{p-1}+\left.\left|\mathbb{D} u-(\mathbb{D} u)_{z_{0}}\right|\right|^{p} \\
& \leq C C_{(5.21)}^{p}\left(\left|(u)_{z_{0}}\right|+\left|(\mathbb{D} u)_{z_{0}}\right|\right) \varrho^{p}\left[\varrho^{p \beta}+\left(f_{Q_{\varrho}\left(z_{0}\right)}\left|u-(u)_{z_{0}}\right|^{p}\right)^{\beta}\right. \\
& \left.+f_{Q_{\varrho}\left(z_{0}\right)}\left|u-(u)_{z_{0}}\right|^{p} \max _{r=1, p-1}\left(f_{Q_{\varrho}\left(z_{0}\right)}\left|\mathbb{D} u-(\mathbb{D} u)_{z_{0}}\right|^{p}\right)^{r}\right]
\end{aligned}
$$

Summing up, (6.27) takes the form 


$$
\begin{aligned}
& \int_{Q_{\varrho}\left(z_{0}\right)}\left|u-(u)_{z_{0}}-(\nabla u)_{z_{0}}\left(x-x_{0}\right)\right|^{p} \leq C \varrho^{p} \underset{Q_{\varrho}\left(z_{0}\right)}{f}\left|\nabla u-(\nabla u)_{z_{0}}\right|^{p} \\
& +C \varrho^{p} C_{(5.21)}^{p}\left(\left|(u)_{z_{0}}\right|+\left|(\mathbb{D} u)_{z_{0}}\right|\right)\left[\varrho^{p \beta}+\max _{l=1, \beta}\left(\underset{Q_{\varrho}\left(z_{0}\right)}{f}\left|u-(u)_{z_{0}}\right|^{p}\right)^{l}\right. \\
& \left.+\max _{r=1, p-1}\left(f_{Q_{\varrho}\left(z_{0}\right)}\left|\mathbb{D} u-(\mathbb{D} u)_{z_{0}}\right|^{p}\right)^{r}\right]
\end{aligned}
$$

Further, estimate

$$
\begin{aligned}
& f_{Q_{\varrho}\left(z_{0}\right)}\left|u-(u)_{z_{0}}\right|^{p} \leq C \int_{Q_{\varrho}\left(z_{0}\right)}\left[\left|u-(u)_{\eta, x_{0}}(t)\right|^{p}+\left|(u)_{\eta, x_{0}}(t)-(u)_{\eta, z_{0}}\right|^{p}\right] d x d t \\
& +C \int_{t_{0}-\varrho^{2}}^{t_{0}}\left|(u)_{\eta, x_{0}}(t)-(u)_{x_{0}}(t)\right|^{p} d t \\
& \leq C \varrho^{p} f_{Q_{\varrho}\left(z_{0}\right)}|\nabla u|^{p}+C \int_{t_{0}-\varrho^{2}}^{t_{0}} f_{t_{0}-\varrho^{2}}^{t_{0}}\left|\int_{B_{\varrho}\left(x_{0}\right)}(u(t, x)-u(r, x)) \eta_{\varrho}(x) d x\right|^{p} d r d t \\
& +C f_{Q_{\varrho}\left(z_{0}\right)}\left|u-(u)_{\eta, x_{0}}(t)-(\nabla u)_{z_{0}}\left(x-x_{0}\right)\right|^{p}+\left|u-(u)_{x_{0}}(t)-(\nabla u)_{z_{0}}\left(x-x_{0}\right)\right|^{p} d t \\
& \leq C \varrho^{p}\left(f_{Q_{\varrho}\left(z_{0}\right)}|\nabla u|^{p}+C_{(5.22)}^{p}\left(\left|(u)_{z 0}\right|+\left|(\mathbb{D} u)_{z_{0}}\right|\right)\left[f_{Q_{\varrho}\left(z_{0}\right)} 1+|\mathbb{D} u|^{p-1}\right]^{p}\right) \\
& +C \varrho^{p} f_{Q_{\varrho}\left(z_{0}\right)}\left|\nabla u-(\nabla u)_{z_{0}}\right|^{p} \\
& \leq C\left(\left|(u)_{z_{0}}\right|+\left|(\nabla u)_{z_{0}}\right|\right) \varrho^{p}\left(1+\left(f_{Q_{\varrho}\left(z_{0}\right)}\left|\mathbb{D} u-(\mathbb{D} u)_{z_{0}}\right|^{p}\right)^{p-1}\right) \\
& +C\left(\left|(u)_{z_{0}}\right|+\left|(\nabla u)_{z_{0}}\right|\right) \varrho^{p} f_{Q_{\varrho}\left(z_{0}\right)}\left|\nabla u-(\nabla u)_{z_{0}}\right|^{p}
\end{aligned}
$$

where for the second inequality we use Poincaré in space and computation analogous to that of (6.29) and for the third one: estimate (5.22) and again Poincaré (both for standard and generalized integral means). (6.31) is (6.24); used in (6.30), it gives 


$$
\begin{aligned}
& \varrho^{-p} f_{Q_{\varrho}\left(z_{0}\right)}\left|u-(u)_{z_{0}}-(\nabla u)_{z_{0}}\left(x-x_{0}\right)\right|^{p} \leq C \underset{Q_{\varrho}\left(z_{0}\right)}{f}\left|\nabla u-(\nabla u)_{z_{0}}\right|^{p} \\
& +C\left(\left|(u)_{z_{0}}\right|+\left|(\nabla u)_{z_{0}}\right|\right)\left[\varrho^{p \beta}+\max _{l=1, \beta}\left(\varrho^{p}+f_{Q_{\varrho}\left(z_{0}\right)}^{f}\left|\nabla u-(\nabla u)_{z_{0}}\right|^{p}\right)^{l}\right] \\
& +C\left(\left|(u)_{z_{0}}\right|+\left|(\nabla u)_{z_{0}}\right|\right)\left[\max _{r=1, \beta(p-1), p-1}\left(f_{Q_{\varrho}\left(z_{0}\right)}\left|\mathbb{D} u-(\mathbb{D} u)_{z_{0}}\right|^{p}\right)^{r}\right]^{(6.32)}
\end{aligned}
$$

which is (6.23). Finally to obtain (6.23) from (6.23), estimate from below 1.h.s. of (6.27), using first (4.5), then the minimization property of $l_{\varrho}^{(p)}$

$$
\begin{aligned}
& f_{Q_{\varrho}\left(z_{0}\right)}\left|u-l_{\varrho}^{(2)}\right|^{p} \leq 2^{p-1}\left(C_{(4.5)}(n, p)+1\right) f_{Q_{\varrho}\left(z_{0}\right)}\left|u-l_{\varrho}^{(p)}\right|^{p} \\
& \leq C \underset{Q_{\varrho}\left(z_{0}\right)}{f}\left|u-(u)_{\eta, z_{0}}-(\nabla u)_{z_{0}}\left(x-x_{0}\right)\right|^{p}
\end{aligned}
$$

Lemma 6.3. Let $p \geq 2$ and $u \in C\left(-T, 0 ; L^{2}(\Omega)\right) \cap L^{p}\left(-T, 0 ; W^{1, p}(\Omega)\right)$ be a weak solution to (1.1) under structure conditions $(2.1 a-2.2 d)$. Take $z_{0} \in Q_{\varrho}\left(z_{0}\right) \subset$ $Q$ such that

$$
\begin{gathered}
\liminf _{\varrho \rightarrow 0} f_{Q_{\varrho}\left(z_{0}\right)}\left|\nabla u-(\nabla u)_{z_{0}}\right|^{p}=0, \\
\limsup _{\varrho \rightarrow 0}\left|(u)_{z_{0}, \varrho}\right|+\left|(\nabla u)_{z_{0}, \varrho}\right|<+\infty
\end{gathered}
$$

then in $\tilde{Q}\left(z_{0}\right)$, denoting a certain vicinity of $z_{0}$, holds

$$
\nabla u \in C^{\beta, \frac{\beta}{2}}\left(\tilde{Q}\left(z_{0}\right)\right)
$$

where $\beta$ is given by (2.1d).

Proof. In view of Lemma $4.2(u)_{z_{0}, \varrho}=l_{\varrho}^{(2)}\left(x_{0}\right)$; this, assumptions (6.34), (6.35) and pointwise estimate $|\mathbb{D} g| \leq|\nabla g|$ imply that we can find sequence $\varrho_{n} \rightarrow 0$ for which the following hold for a certain $M<\infty$

$$
\begin{array}{r}
\lim _{\varrho_{n} \rightarrow 0}\left[f_{Q_{\varrho_{n}}\left(z_{0}\right)}\left|\mathbb{D} u-(\mathbb{D} u)_{z_{0}}\right|^{p}+\int_{Q_{\varrho_{n}}\left(z_{0}\right)}\left|\nabla u-(\nabla u)_{z_{0}}\right|^{p}\right] \\
\left|(\nabla u)_{z_{0}, \varrho_{n}}\right| \leq M / 8, \quad\left|l_{\varrho_{n}}^{(2)}\left(x_{0}\right)\right| \leq M / 8, \quad \tilde{E}_{l_{\ell n}^{(2)}} \rightarrow 0
\end{array}
$$


where Hölder inequality and $p \geq 2$ is used to control $\psi_{2}$ in $\tilde{E}$ with $\psi_{p}$ being 1.h.s. of (6.23). Moreover (6.37), (6.38) with inequality (6.23) give again via Hölder inequality

$$
\lim _{\varrho_{n} \rightarrow 0} \varrho_{n}^{-2} f_{Q_{\varrho_{n}}\left(z_{0}\right)}\left|u-(u)_{z_{0}, \varrho_{n}}-(\nabla u)_{z_{0}, \varrho_{n}}\left(x-x_{0}\right)\right|^{2}=0
$$

In order to replace $\left|(\nabla u)_{z_{0}, \varrho}\right|$ in (6.38) with $\left|\nabla l_{z_{0}, \varrho_{n}}^{(2)}\right|$, perform estimate using inequality (4.3) of Lemma 4.2

$$
\left|\nabla l_{z_{0}, \varrho_{n}}^{(2)}\right|^{2} \leq \frac{2 d(d+2)}{\varrho_{n}^{2}} f_{Q_{\varrho_{n}}\left(z_{0}\right)}\left|u-(u)_{z_{0}, \varrho_{n}}-(\nabla u)_{z_{0}, \varrho_{n}}\left(x-x_{0}\right)\right|^{2}+\left|(\nabla u)_{z_{0}, \varrho_{n}}\right|^{2},
$$

which in view of (6.39) and (6.40) gives for $n \geq n_{0}$

$$
\left|\nabla l_{z_{0}, \varrho_{n}}^{(2)}\right| \leq M / 4
$$

This and (6.38) shows that there is a sequence $\varrho_{m} \rightarrow 0\left(m=n-n_{0}\right)$ for which holds for a certain $M<\infty$

$$
\left|\nabla l_{z_{0}, \varrho_{m}}^{(2)}\right| \leq M / 4, \quad\left|l_{\varrho_{m}}^{(2)}\left(x_{0}\right)\right| \leq M / 4, \quad \tilde{E}_{l_{\varrho m}^{(2)}} \rightarrow 0
$$

Constant $M$ and choice $\alpha \in(\beta, 1)$, fixes $\sigma \in(0,1 / 4), \delta \in(0,1)$ via Lemma 6.1. Convergences in (6.42) imply the existence of such $\varrho_{0}$ that

$$
\omega\left(M+1, \tilde{E}_{l_{z_{0}, \varrho_{0}}^{(2)}}\left(\varrho_{0}\right)\right)+\tilde{E}_{l_{z_{0}, \varrho_{0}}^{(2)}}^{\frac{1}{2}}\left(\varrho_{0}\right)<\frac{\delta}{2 C_{5.4}(M)}
$$

holds; in fact, for this fixed radius $\varrho_{0}$, the absolute continuity of integrals with respect to the Lebesgue measure and a continuity of the modulus of continuity $\omega$ imply that we have for any point of $\tilde{z} \in \tilde{Q}\left(z_{0}\right), \tilde{Q}\left(z_{0}\right)$ being a certain neighborhood of $z_{0}$,

$$
\begin{aligned}
& \left|\nabla l_{\tilde{z}, \varrho_{m}}^{(2)}\right| \leq M / 4, \quad\left|l_{\tilde{z}, \varrho_{m}}^{(2)}(\tilde{x})\right| \leq M / 4, \\
& \omega\left(M+1, \tilde{E}_{l_{\tilde{z}, \varrho_{0}}^{(2)}}\left(\varrho_{0}\right)\right)+\tilde{E}_{l_{\tilde{z}, \varrho_{0}}^{(2)}}^{\frac{1}{2}}\left(\varrho_{0}\right)<\frac{\delta}{2 C_{5.4}(M)}
\end{aligned}
$$

in what follows, we generally abandon the dependence of the following expressions on $\tilde{z}$, remembering that it is an arbitrary point from $\tilde{Q}\left(z_{0}\right)$. Lemma 6.1 and (6.44) give

$$
\tilde{E}_{l_{\sigma \varrho_{0}}^{(2)}}\left(\sigma \varrho_{0}\right) \leq \sigma^{2 \beta} \tilde{E}_{l_{\varrho_{0}}^{(2)}}\left(\varrho_{0}\right)+C(M, \beta)\left(\sigma \varrho_{0}\right)^{2 \beta}
$$

The next step is to prove that for every $j \in \mathbb{N}$ holds

$$
\tilde{E}_{l_{\sigma^{j} \varrho_{0}}^{(2)}}\left(\sigma^{j} \varrho_{0}\right) \leq \sigma^{j 2 \beta} \tilde{E}_{l_{\varrho_{0}}^{(2)}}\left(\varrho_{0}\right)+C(M, \beta)\left(\sigma^{j} \varrho_{0}\right)^{2 \beta}, \quad\left|l_{\sigma^{j} \varrho_{0}}^{(2)}\right|+\left|\nabla l_{\sigma^{j} \varrho_{0}}^{(2)}\right| \leq M
$$


as the inductive argument here is identical as the respective part of the proof of Lemma 4.9 in [10], we do not present it here.

Now we show, for the above fixed $\varrho_{0}, \sigma, M$, that

$$
\lim _{j \rightarrow \infty}(\nabla u)_{\tilde{z}, \sigma^{j} \varrho_{0}} \equiv \tilde{\Gamma}
$$

exists and for $r \in\left(0, \varrho_{0} / 2\right)$

$$
f_{Q_{r}(\tilde{z})}|\nabla u-\tilde{\Gamma}|^{2} \leq C r^{2 \beta}
$$

Fix $r \in\left(0, \varrho_{0} / 2\right)$ and choose $j$ such that

$$
\sigma^{j+1}\left(\varrho_{0} / 2\right)<r \leq \sigma^{j}\left(\varrho_{0} / 2\right)
$$

Then by the minimizing property of a mean value (the first inequality), Caccioppoli inequality (5.24) (the middle inequality), (6.46) (the third one) we get

$$
\begin{aligned}
& \underset{Q_{r}}{f}\left|\nabla u-(\nabla u)_{Q_{r}}\right|^{2} \\
& \quad \leq(1 / \sigma)^{n+2} f_{Q_{\sigma^{j}\left(\varrho_{0} / 2\right)}}\left|\nabla u-\nabla l_{\sigma^{j}\left(\varrho_{0} / 2\right)}^{(2)}\right|^{2} \leq(1 / \sigma)^{n+2} \tilde{E}_{l_{\sigma^{j} \varrho_{0}}^{(2)}}\left(\sigma^{j} \varrho_{0}\right) \\
& \leq(1 / \sigma)^{n+2}\left[\sigma^{j 2 \beta} \tilde{E}_{l_{\varrho_{0}}^{(2)}}\left(\varrho_{0}\right)+C(M, \beta)\left(\sigma^{j} \varrho_{0}\right)^{2 \beta}\right] \\
& \leq(1 / \sigma)^{n+2}\left[\tilde{E}_{\left.l_{\varrho_{0}}^{(2)}\left(\varrho_{0}\right)+C(M, \beta) \varrho_{0}^{2 \beta}\right] \sigma^{j 2 \beta} \leq C(M, \beta) r^{2 \beta}}\right.
\end{aligned}
$$

Similarly for $j<k$

$$
\begin{aligned}
& \left|(\nabla u)_{\sigma^{j}(\varrho / 2)}-(\nabla u)_{\sigma^{k}(\varrho / 2)}\right| \leq \sum_{m=j+1}^{k}\left|(\nabla u)_{\sigma^{m}(\varrho / 2)}-(\nabla u)_{\sigma^{m-1}(\varrho / 2)}\right| \\
& \leq \sum_{m=j+1}^{k}\left|f_{Q_{\sigma^{m}(\varrho / 2)}} \nabla u-(\nabla u)_{\sigma^{m-1}(\varrho / 2)}\right| \\
& \leq(1 / \sigma)^{\frac{n+2}{2}} \sum_{m=j+1}^{k}\left[\int_{Q_{\sigma}^{m-1}(\varrho / 2)}\left|\nabla u-(\nabla u)_{\sigma^{m-1}(\varrho / 2)}\right|^{2}\right]^{\frac{1}{2}} \\
& \leq(1 / \sigma)^{\frac{n+2}{2}}\left[\tilde{E}_{l_{\varrho 0}^{(2)}}\left(\varrho_{0}\right)+C(M, \beta) \varrho_{0}^{2 \beta}\right]^{1 / 2} \sum_{m=j}^{k-1} \sigma^{m \beta} \\
& \leq(1 / \sigma)^{\frac{n+2}{2}}(1-\sigma)^{-1} C^{1 / 2}(M, \beta) \sigma^{\beta j}=\tilde{C}(M, \beta) \sigma^{\beta j}
\end{aligned}
$$


where the last but one inequality is obtained as (6.50). The estimate (6.51) states that $\lim _{j \rightarrow \infty}(\nabla u)_{\sigma^{j}} \frac{\varrho}{2}=\tilde{\Gamma}$ exists and that

$$
\left|(\nabla u)_{\tilde{z}, \sigma^{j}(\varrho / 2)}-\tilde{\Gamma}\right| \leq \tilde{C}(M, \beta) \sigma^{\beta j}
$$

This combined with (6.50) results in:

$$
\begin{aligned}
& \int_{Q_{r}}|\nabla u-\tilde{\Gamma}|^{2} \leq(1 / \sigma)^{n+2} \underset{Q_{\sigma^{j}\left(\varrho_{0} / 2\right)}}{f}\left|\nabla u \pm(\nabla u)_{\sigma^{j}\left(\varrho_{0} / 2\right)}-\tilde{\Gamma}\right|^{2} \\
& \quad \leq 2\left(\tilde{C}^{2}(M, \beta)+C(M, \beta)\right) \sigma^{2 \beta j} \leq C(M, \beta) r^{2 \beta}
\end{aligned}
$$

where the last inequality holds in view of (6.49). As (6.53) is valid for any $\tilde{z}$, being an arbitrary point from $\tilde{Q}\left(z_{0}\right)$, imbedding of Campanato into Hölder spaces gives

$$
\nabla u \in C^{\beta, \frac{\beta}{2}}\left(\tilde{Q}\left(z_{0}\right)\right) .
$$

We are done with the partial regularity result for the gradient. Let us now focus on an analogous property for the solution itself, stated in the following result.

Lemma 6.4. Take $\tilde{Q}\left(z_{0}\right)$ - a neighborhood of a regular point of Lemma 6.3. Under assumptions of Lemma 6.3 holds

$$
u \in C^{1, \frac{1}{2}}\left(\tilde{Q}\left(z_{0}\right)\right) \text {. }
$$

Proof. For any $x, y \in B_{\tilde{\varrho}}(\tilde{x}) \subset \tilde{Q}\left(z_{0}\right)$ the following pointwise estimate for a weak solution $u$ to (1.1), $u \in C\left(-T, 0 ; L^{2}(\Omega)\right) \cap L^{p}\left(-T, 0 ; W^{1, p}(\Omega)\right)$ holds

$$
\begin{aligned}
& \left|\int_{B_{\tilde{\varrho}}(\tilde{x})}(u(\tilde{\tau}, x)-u(\tilde{\tau}, y)) \eta_{\varrho}(x) d x\right| \\
& \leq C f_{B_{\tilde{Q}}(\tilde{x})}(M(\nabla u)(\tilde{\tau}, x)+M(\nabla u)(\tilde{\tau}, y))|x-y| d x \leq C_{\tilde{Q}\left(z_{0}\right)} \tilde{\varrho},
\end{aligned}
$$

where $M(f)$ is a maximal function. The first inequality is given by Bojarski-Hajłasz inequality (see [3], Theorem 3) and the second is a consequence of boundedness of gradients given by Lemma 6.3. Adding (5.22), which holds for every time level, and twice (6.55): the first one with $\tilde{\tau}=\tau, y=y_{1}$ and the second one with $\tilde{\tau}=t, y=y_{2}$ we obtain thanks to boundedness of $\nabla u$

$$
\begin{aligned}
& C_{\tilde{Q}\left(z_{0}\right)} \tilde{\varrho} \geq\left|\int_{B_{\tilde{\varrho}}(\tilde{x})}(u(t, x)-u(\tau, x)) \eta_{\varrho}(x) d x\right|+\left|\int_{B_{\tilde{\varrho}}(\tilde{x})}\left(u(t, x)-u\left(t, y_{1}\right)\right) \eta_{\varrho}(x) d x\right| \\
& \quad+\left|\int_{B_{\tilde{\varrho}}(\tilde{x})}\left(u(\tau, x)-u\left(\tau, y_{2}\right)\right) \eta_{\varrho}(x) d x\right| \geq\left|u\left(t, y_{1}\right)-u\left(\tau, y_{2}\right)\right|
\end{aligned}
$$

which gives thesis. 
Finally we see that

Proof (of Theorem 2.1). results from Lemmas 6.3 and 6.4.

\section{Appendix}

Here we present results which have been removed from the main part of this article for the sake of traceability.

Proof (of Korn's inequality (4.6b) in Lemma 4.3). Use inequality from [6]

$$
K \int_{B_{1}(x)}|\mathbb{D} h|^{2} \geq \inf _{R \in \mathcal{R}} \int_{B_{1}(x)}|\nabla[h-R]|^{2}
$$

where $\mathcal{R}$ is the set of rigid motions, i.e. affine functions with antisymmetric linear part. (7.1) with $h:=u-(\mathbb{D} u)\left(x-x_{0}\right)$ yields:

$$
K \int_{B_{1}(x)}|\mathbb{D} u-(\mathbb{D} u)|^{2} \geq \inf _{R \in \mathcal{R}} \int_{B_{1}(x)}|\nabla u-(\mathbb{D} u)-R|^{2} \geq \int_{B_{1}(x)}|\nabla u-(\nabla u)|^{2}
$$

we have also pointwisely $|\nabla h|^{2} \geq|\mathbb{D} h|^{2}$, so for $h:=u-(\nabla u)\left(x-x_{0}\right)$ it gives

$$
\int_{B_{1}(x)}|\nabla u-(\nabla u)|^{2} \geq \int_{B_{1}(x)}|\mathbb{D} u-(\mathbb{D} u)|^{2}
$$

The independence of $K$ on radius $r$ comes from scaling.

Let us remark that the Korn's inequality can be traced back to the papers of Friederichs [12] and Korn himself [14]. Next we show the needed result on linear systems.

Proof (of Lemma 4.4). Smoothness is a standard result for systems with coefficients depending on full gradient and satisfying Legendre-Hadamard conditions. See [11], [18]. To prove inequalities we modify slightly the technique of Campanato [5]. Scaling $v(y, s)=u\left(r y, r^{2} s\right)$ justifies that $u$ solves locally (4.7) in $Q_{r}$ iff $v$ solves (4.7) locally in $Q_{1}$. Therefore we consider first $v$ satisfying

$$
\int_{Q_{1}} v \varphi_{, t}-A \mathbb{D} v \mathbb{D} \phi=0 \quad \underset{\varphi \in C_{0}^{\infty}\left(Q_{1}\right)}{\forall}
$$

Take a smooth cutoff functions from $C_{0}^{\infty}\left(Q_{1}\right)$ that satisfies

$$
\begin{gathered}
{[0,1] \ni \theta(x)=\left\{\begin{array}{ll}
1 & B_{\frac{1}{2}} \\
0 & B_{1}^{c}
\end{array} \quad|\nabla \theta| \leq 8\right.} \\
{[0,1] \ni \sigma(t)= \begin{cases}1 & t \in\left(\frac{1}{4}, 0\right), \quad\left|\sigma_{, t}\right| \leq 8 \\
0 & t \leq 1\end{cases} }
\end{gathered}
$$


we test (4.9) with $\theta^{2} \sigma^{2} v$, which yields:

(i) for the main part:

$$
\begin{aligned}
& \frac{1}{2} \sigma^{2} a_{k l}^{i j}\left(v_{l}^{k}+v_{k}^{l}\right)\left(\theta^{2} v^{i}\right)_{, j}=\frac{1}{2} a_{l}^{i j}\left(v_{l}^{k}+v_{k}^{l}\right)\left[\theta\left(\theta v^{i}\right)_{, j}+\theta, j \theta v^{i}\right] \\
& =\frac{\sigma^{2}}{2} a_{k l}^{i j}\left[(v \theta)_{, l}^{k}+(v \theta)_{, k}^{l}\right]\left(\theta v^{i}\right)_{, j}-\frac{\sigma^{2}}{2} a_{k l}^{i j}\left(v \theta_{, l}+v \theta_{, k}\right)\left(\theta v^{i}\right)_{, j} \\
& \quad+\frac{\sigma^{2}}{2} a_{k l}^{i j}\left(v_{l}^{k}+v_{k}^{l}\right) \theta v^{i} \theta_{, j} \geq \lambda|\mathbb{D}(v \sigma \theta)|^{2}+\frac{\sigma^{2} \theta}{2}\left[a_{k l}^{i j}\left(v_{l}^{k}+v_{k}^{l}\right) v^{i} \theta_{, j}\right. \\
& \left.\quad-a_{k l}^{i j}\left(v^{k} \theta_{, l}+v^{l} \theta_{, k}\right) v_{, j}^{i}\right]-|A|\left|\sigma^{2}\right||\nabla \theta|^{2}|v|^{2}
\end{aligned}
$$

and the middle part vanishes because $a_{k l}^{i j}=a_{i j}^{k l}=a_{l k}^{j i}$

$$
\begin{gathered}
a_{k l}^{i j} v_{l}^{k} v^{i} \theta_{, j}+a_{k l}^{i j} v_{k}^{l} v^{i} \theta_{, j}-\overbrace{a_{k l}^{i j} v_{j}^{i} v^{k} \theta_{, l}}^{a_{i j}^{k l} v_{l}^{k} v^{i} \theta_{, j}}=\overbrace{a_{k l}^{i j} v_{j}^{i} v^{l} \theta_{, k}}^{=a_{j i}^{l k} v_{k}^{l} v^{i} \theta_{, j}} \\
=\underbrace{\left(a_{k l}^{i j}-a_{i j}^{k l}\right)}_{=0} v_{, l}^{k} v^{i} \theta_{, j}+\underbrace{\left(a_{k l}^{i j}-a_{l k}^{j i}\right)}_{=0} v_{k}^{l} v^{i} \theta_{, j}
\end{gathered}
$$

(ii) for the evolutionary part:

$$
v_{, t} \theta^{2} \sigma^{2} v=\frac{1}{2}\left(v^{2} \theta^{2} \sigma^{2}\right)_{, t}-v^{2} \theta^{2} \sigma_{, t} \sigma
$$

By (7.3) and (7.5) integrated over $Q_{1}$ we have:

$$
\frac{1}{2} \frac{d}{d t} \int_{Q_{1}} v^{2} \theta^{2} \sigma^{2}+\lambda \int_{Q_{1}}|\mathbb{D}(v \theta \sigma)|^{2} \leq 8|A| \int_{Q_{1}}|v|^{2}
$$

as $\frac{d}{d t} \int_{Q_{1}} v^{2} \theta^{2} \sigma^{2}=\int_{B_{1}} v^{2}(x, 0) \theta^{2}(x) d x \geq 0$, we have from (7.6) and Korn's inequality

$$
\int_{Q_{1 / 2}}|\nabla v|^{2} \leq C\left[\int_{Q_{1 / 2}}|\mathbb{D} v|^{2}+\int_{Q_{1 / 2}}|v|^{2}\right] \leq C \int_{Q_{1}}|v|^{2}
$$

with constant $C$ depending on $\lambda,|A|, C_{K o r_{p}}$. By linearity of (7.2) arbitrary derivative of $v$ satisfies again (7.2). Because the time derivative can be expressed by space derivative via equation, one can iterate (7.7) obtaining (with a slight abuse of cutoff function, which should have been stated for $Q_{1-2^{-n}}$ )

$$
\int_{Q_{1 / 2}}\left|\nabla^{(2 k)} v\right|^{2}+\left|\partial_{t}^{(k)} v\right|^{2} \leq C\left(\lambda,|A|, C_{K o r_{p}}, k\right)|v|_{L^{2}\left(Q_{1}\right)}^{2}
$$

which from Sobolev imbedding, for $k$ big enough, gives

$$
\sup _{Q_{1 / 2}}\left|\nabla^{(2 m)} v\right|^{2}+\left|\partial_{t}^{(m)} v\right|^{2} \leq C\left(\lambda,|A|, C_{K o r_{p}}, m, n\right)|v|_{L^{2}\left(Q_{1}\right)}^{2}
$$


and as a result for arbitrary $q \geq 1$ and $\rho / r \leq 1 / 2$ both

$$
\left[f_{Q_{\rho / r}}\left|\nabla^{(2 m)} v\right|^{q}+\left|\partial_{t}^{(m)} v\right|^{q}\right]^{\frac{1}{q}} \leq C\left(\lambda,|A|, C_{K o r_{p}}, m, n\right)|v|_{L^{2}\left(Q_{1}\right)}
$$

and

$$
\begin{aligned}
& {\left[f_{Q_{\rho / r}}\left|v^{(m-1)}-v^{(m-1)}\left(\tilde{z}_{0}\right)\right|^{q}\right]^{\frac{1}{q}} \leq\left(\frac{\varrho}{r}\right) C\left(\lambda,|A|, C_{K o r_{p}}, m, n\right)|v|_{L^{2}\left(Q_{1}\right)}} \\
& {\left[f_{Q_{\rho / r}}\left|v^{(m-1)}-\left(v^{(m-1)}\right)_{Q_{\rho / r}}\right|^{q} \leq\left(\frac{\varrho}{r}\right) C\left(\lambda,|A|, C_{K o r_{p}}, m, n\right)|v|_{L^{2}\left(Q_{1}\right)}^{2}\right.}
\end{aligned}
$$

hold, where $\tilde{z}_{0}$ is an arbitrary point, $v^{(m)}$ denotes $\left(\nabla^{(2 m)} v, \partial_{t}^{(m)} v\right)$. To obtain the last two inequalities one uses

$$
\left[f_{Q_{\rho / r}}\left|h-h\left(\tilde{z}_{0}\right)\right|^{q}\right]^{\frac{1}{q}} \leq\left(\frac{\varrho}{r}\right) \sup _{Q_{\rho / r}}\left(|\nabla h|+\left|h_{, t}\right|\right)
$$

Rescaling inequalities (7.11)-(7.13) from $v$ back to $u$ and shifting $m-1$ to $m$ in (7.11), (7.12) we obtain

$$
\begin{aligned}
& {\left[\int_{Q_{\rho}}\left|\nabla^{(2 m)} u\right|^{q}+\left|\partial_{t}^{(m)} u\right|^{q}\right]^{\frac{1}{q}} \leq r^{-2 m} C\left(\lambda,|A|, C_{K o r_{p}}, m, n\right)\left[f|u|^{2}\right]_{Q_{r}}^{1 / 2}} \\
& \left.\left[f_{Q_{\rho}}\left|u^{(m)}-u^{(m)}\left(\tilde{z}_{0}\right)\right|^{q}\right]^{\frac{1}{q}} \leq r^{-2 m}\left(\frac{\varrho}{r}\right) C\left(\lambda,|A|, C_{K o r_{p}}, m, n\right)\left[f|u|^{2}\right]^{\frac{1}{2}}\right]_{Q_{r}}^{(7.15)} \\
& {\left[f_{Q_{\rho}}\left|u^{(m)}-\left(u^{(m)}\right)_{Q_{\rho}}\right|^{q}\right]^{\frac{1}{q}} \leq r^{-2 m}\left(\frac{\varrho}{r}\right) C\left(\lambda,|A|, C_{K_{o r}}, m, n\right)\left[f|u|^{2}\right]_{Q_{r}}^{\frac{1}{2}}}
\end{aligned}
$$

which together with Giusti's technique, allowing in local inequalities to decrease the power of integrability on right-hand-sides below 2 by interpolation, implies thesis. 
Let us now turn to the proof of symmetric caloric approximation lemma.

Proof (of Lemma 4.5). First consider unit cylinder, i.e. $Q_{\varrho}\left(z_{0}\right)=Q_{1}$. We perform an indirect proof. Contradiction yields existence of $\varepsilon_{0}>0, \lambda_{0}>0, M_{0}>0$ such that for any $k \in \mathbb{N}$ one can find such $A_{k} \in S\left(\lambda_{0}, M_{0}\right), \gamma_{k} \in[0,1]$ that

$$
f_{k} \in H\left(1 ; 1 / k, A_{k}, \gamma_{k}\right) \wedge \underset{h \in H\left(1 / 2 ; A_{k}, \gamma_{k}\right)}{\forall} f_{Q_{1 / 2}} 4\left|h-f_{k}\right|^{2}+\gamma_{k}^{p-2} 2^{p}\left|h-f_{k}\right|^{p} \geq \varepsilon_{0}
$$

By $\gamma_{k} \in[0,1]$, definitions of approximatively weakly symmetrical caloric functions and $S\left(\lambda_{0}, M_{0}\right)$ we have the following convergences for $k \rightarrow \infty$ (up to nonrelabeled subsequence)

$$
\begin{aligned}
\gamma_{k} & \rightarrow \gamma & A_{k} & \rightarrow A \in S\left(\lambda_{0}, M_{0}\right) \\
f_{k} & \rightarrow f \text { in } L^{2}\left(Q_{1}\right) & \nabla f_{k} & \rightarrow \nabla f \text { in } L^{2}\left(Q_{1}\right) \\
\gamma_{k}^{\frac{p-2}{p}} f_{k} & \rightarrow \gamma^{\frac{p-2}{p}} f \text { in } L^{p}\left(Q_{1}\right) & \gamma_{k}^{\frac{p-2}{p}} \nabla f_{k} & \rightarrow \gamma^{\frac{p-2}{p}} \nabla f \text { in } L^{p}\left(Q_{1}\right)
\end{aligned}
$$

The fact that $A$ is symmetrizing comes from $A_{k}$ being symmetrizing. The last two limits can be identified thanks to previous convergences. Consequently by l.w.s.c. of Lebesgue norms one has

$$
f_{Q_{1}}|f|^{2}+|\nabla f|^{2}+\gamma^{p-2}\left[|f|^{p}+|\nabla f|^{p}\right] \leq 1
$$

Write for arbitrary $\varphi \in C_{0}^{\infty}\left(Q_{1}\right)$

$$
\begin{aligned}
& \int_{Q_{1}} f \varphi, t-A(\mathbb{D} f, \mathbb{D} \varphi)=\int_{Q_{1}}\left(f-f_{k}\right) \varphi_{, t}-\int_{Q_{1}} A\left(\mathbb{D} f-\mathbb{D} f_{k}, \mathbb{D} \varphi\right) \\
& -\int_{Q_{1}}\left(A-A_{k}\right)\left(\mathbb{D} f_{k}, \mathbb{D} \varphi\right)+\int_{Q_{1}} f_{k} \varphi_{, t}-A_{k}\left(\mathbb{D} f_{k}, \mathbb{D} \varphi\right)=I+I I+I I I+I V
\end{aligned}
$$

one has that $I, I I, I I I \stackrel{k \rightarrow \infty}{\rightarrow} 0$ by (7.18), IV $\stackrel{k \rightarrow \infty}{\rightarrow} 0$ as $f_{k} \in H\left(1 ; 1 / k, A_{k}, \gamma_{k}\right)$; so

$$
\int_{Q_{1}} f \varphi, t-A(\mathbb{D} f, \mathbb{D} \varphi)=0 \quad \underset{\varphi \in C_{0}^{\infty}\left(Q_{1}\right)}{\forall}
$$

(7.20) and (7.19) imply

$$
f \in H(1 ; A, \gamma)
$$

i.e. limit $f$ is in the set of caloric functions.

Assume for a moment that we have also strong convergences

$$
f_{k} \rightarrow f \text { in } L^{2}\left(Q_{1}\right), \quad \gamma_{k}^{\frac{p-2}{p}} f_{k} \rightarrow \gamma^{\frac{p-2}{p}} f \text { in } L^{p}\left(Q_{1}\right)
$$


then we would have in view of (7.22)

$$
\lim _{k \rightarrow \infty} \int_{Q_{1}}\left|f-f_{k}\right|^{2}+\gamma_{k}^{p-2}\left|f-f_{k}\right|^{p}=0
$$

which with (7.21) is almost a contradiction to (7.17) with an exception, that we require in (7.17) $h \in H\left(1 / 2 ; A_{k}, \gamma_{k}\right)$ and instead have $f \in H(1 ; A, \gamma)$. We compensate this difference by proceeding as follows. Consider the following linear boundary-value problem

$$
\begin{cases}\omega_{, t}^{k}-\operatorname{div} A_{k} \mathbb{D} \omega^{k}=0 & \text { in } Q_{3 / 4} \\ \omega^{k}=f & \text { on } \partial_{\Gamma} Q_{3 / 4}\end{cases}
$$

(7.24) and (7.20) give

$$
\int_{Q_{3 / 4}}\left(\omega^{k}-f\right) \varphi_{, t}-\int_{Q_{3 / 4}} A_{k}\left(\mathbb{D} \omega^{k}-\mathbb{D} f, \mathbb{D} \varphi\right)=\int_{Q_{3 / 4}}\left(A_{k}-A\right)(\mathbb{D} f, \mathbb{D} \varphi)
$$

since by (7.24) $w^{k}$ and $f$ agree on the boundary, we can test (7.25) with $w^{k}-f$ obtaining

$$
\begin{aligned}
& \sup _{t \in\left(-(3 / 4)^{2}, 0\right)}\left|\omega^{k}(t)-f(t)\right|_{L^{2}\left(B_{3 / 4}\right)}^{2}+\int_{B_{3 / 4}} A_{k}\left(\mathbb{D}\left(\omega^{k}-f\right), \mathbb{D}\left(\omega^{k}-f\right)\right) \\
& =\int_{B_{3 / 4}}\left(A_{k}-A\right)\left(\mathbb{D} f, \mathbb{D}\left(\omega^{k}-f\right)\right)
\end{aligned}
$$

which by ellipticity of $A_{k}$ and Korn's inequality gives

$$
\begin{aligned}
& \left|\omega^{k}-f\right|_{L^{2}\left(Q_{3 / 4}\right)}+\left|\nabla\left(\omega^{k}-f\right)\right|_{L^{2}\left(Q_{3 / 4}\right)} \leq C\left[\sup _{t}\left|\omega^{k}(t)-f(t)\right|_{L^{2}\left(B_{3 / 4}\right)}\right. \\
& \left.+\left|\mathbb{D}\left(\omega^{k}-f\right)\right|_{L^{2}\left(Q_{3 / 4}\right)}\right] \leq C\left|A_{k}-A\right| \stackrel{k \rightarrow \infty}{\rightarrow} 0
\end{aligned}
$$

Observe that in view of inequality (4.9) of Lemma 4.4, $\left|\omega^{k}\right|_{L^{2}\left(Q_{3 / 4}\right)}$ controls norms on $Q_{1 / 2}$ of $\omega^{k}$ of arbitrary high order. This and (7.26) yield

$$
\left|\left(\omega^{k}-f\right)\right|_{L^{p}\left(Q_{1 / 2}\right)}+\left|\nabla\left(\omega^{k}-f\right)\right|_{L^{p}\left(Q_{1 / 2}\right)} \rightarrow 0
$$

We show now that $\omega_{k}$ contradicts (7.17) for large $k$. One has

$$
f_{Q_{1 / 2}} \gamma_{k}^{p-2}\left|\omega_{k}-f_{k}\right|^{p} \leq f_{Q_{1 / 2}}\left|\gamma_{k}^{p-2} f-\gamma_{k}^{p-2} f_{k}\right|^{p}+f_{Q_{1 / 2}}\left|\omega_{k}-f\right|^{p} \rightarrow 0
$$

where the convergence stems from (7.27) and (7.22). Similarly

$$
\int_{Q_{1 / 2}}\left|\omega_{k}-f_{k}\right|^{2} \rightarrow 0
$$


What's more, $\gamma_{k} \rightarrow \gamma,(7.26)$ and (7.27) imply

$$
\begin{aligned}
& \int_{Q_{1 / 2}}\left|\omega_{k}\right|^{2}+\left|\nabla \omega_{k}\right|^{2}+\gamma_{k}^{p-2}\left[\left|\omega_{k}\right|^{p}+\left|\nabla \omega_{k}\right|^{p}\right] \\
& \quad \rightarrow f_{Q_{1 / 2}}|f|^{2}+|\nabla f|^{2}+\gamma^{p-2}\left[|f|^{p}+|\nabla f|^{p}\right] \leq 2^{n+2}
\end{aligned}
$$

where the last inequality holds thanks to $f \in H(1 ; A, \gamma)$. (7.30) and (7.24) state that $\omega_{k} \in H\left(1 / 2 ; A_{k}, \gamma_{k}\right)$ for large $k$, whence (7.28), (7.29) show that $\omega_{k}$ approximates $f_{k}$ in $L^{2}-L^{p}$ sense. This contradicts (7.17). Thus we are done with the case of an unit parabolic cylinder, provided (7.22) really holds, which now will be proven by means of parabolic compactness of Lemma 4.1. By (7.17) $f_{k} \in H\left(1 ; 1 / k, A_{k}, \gamma_{k}\right)$, so from its definition

$$
\left|\int_{Q} \gamma_{k}^{\frac{p-2}{p}} f_{k} \varphi_{, t}\right| \leq \lambda_{0}^{-1}\left(\int_{Q}|\mathbb{D} \varphi|^{p^{\prime}}\right)^{\frac{1}{p^{\prime}}}+\frac{1}{k} \sup _{Q}|\mathbb{D} \varphi|
$$

taking $\varphi=\xi(x) \eta_{s_{1}, s_{2}}^{\varepsilon}(t)$ with

$$
\begin{gathered}
\eta= \begin{cases}1 & t \in\left[s_{1}, s_{2}\right] \\
0 & t \notin\left[s_{1}-\varepsilon, s_{2}+\varepsilon\right] \\
\text { affine } & \text { otherwise }\end{cases} \\
\left|\int_{B} \frac{1}{\varepsilon}\left[\int_{s_{1}-\varepsilon}^{s_{1}} \gamma_{k}^{\frac{p-2}{p}} f_{k}-\int_{s_{2}}^{s_{2}+\varepsilon} \gamma_{k}^{\frac{p-2}{p}} f_{k}\right] \xi\right| \leq\left[\lambda_{0}^{-1}\left(s_{2}-s_{1}+2 \varepsilon\right)^{\frac{1}{p^{\prime}}}+\frac{1}{k}\right]|\mathbb{D} \xi|_{L^{\infty}(B)} \\
\text { so for a.e. } s_{1}, s_{2}, \text { by } \varepsilon \rightarrow 0, \\
\left|\int_{B}\left(\gamma_{k}^{\frac{p-2}{p}} f_{k}\left(\cdot, s_{2}\right)-\gamma_{k}^{\frac{p-2}{p}} f_{k}\left(\cdot, s_{1}\right)\right) \xi\right| \leq\left(c\left(s_{2}-s_{1}\right)^{\frac{1}{p^{\prime}}}+\frac{1}{k}\right)|\nabla \xi|_{L^{\infty}(B)}
\end{gathered}
$$

for $l-\frac{n}{2}>1$ holds $C|\xi|_{W^{l, 2}} \geq|\nabla \xi|_{L^{\infty}}$ so (7.31) and a density argument imply

$$
\left|\gamma_{k}^{\frac{p-2}{p}} f_{k}\left(\cdot, s_{2}\right)-\gamma_{k}^{\frac{p-2}{p}} f_{k}\left(\cdot, s_{1}\right)\right|_{W^{-l, 2}(B)} \leq c\left(\left(s_{2}-s_{1}\right)^{\frac{1}{p^{\prime}}}+\frac{1}{k}\right)
$$

i.e.

$$
\int_{-1}^{-h}\left|\gamma_{k}^{\frac{p-2}{p}} f_{k}(\cdot, t+h)-\gamma_{k}^{\frac{p-2}{p}} f_{k}(\cdot, t)\right|_{W^{-l, 2}(B)}^{p} \leq c\left(h^{p-1}+\frac{1}{k^{p^{\prime}}}\right)
$$


Fix $\varepsilon>0$. For $k>(2 c / \varepsilon)^{\frac{1}{p^{\prime}}}$ r.h.s. of (7.32) does not exceed $\varepsilon$ for every $h \leq$ $(\varepsilon / 2 c)^{\frac{1}{p-1}}$. For a finite number of initial $k$ one can majorize 1.h.s. of (7.32) by $\varepsilon$ for every $h \leq h_{0}$ by properties of Bochner spaces. All in all it holds

$$
\underset{\varepsilon>0}{\forall} \underset{h^{\prime}}{\exists} \underset{h \leq h^{\prime}}{\forall} \int_{-1}^{-h}\left|\gamma_{k}^{\frac{p-2}{p}} f_{k}(\cdot, t+h)-\gamma_{k}^{\frac{p-2}{p}} f_{k}(\cdot, t)\right|_{W^{-l, 2}(B)}^{p} \leq \varepsilon
$$

This and uniform boundedness of $\gamma_{k}^{\frac{p-2}{p}} f_{k}$ in $L^{p}\left(W^{1, p}\right)$ allows us to use Lemma 4.1 with choices $X=W^{1, p}, Y=L^{p}, Z=W^{-l, 2}$ to get, up to subsequence, $\gamma_{k}^{\frac{p-2}{p}} f_{k} \rightarrow \gamma^{\frac{p-2}{p}} f$ in $L^{p}$. Similar argument for $L^{2}$ gives (7.22).

Finally, we perform step from the unit cylinder $Q_{1}\left(z_{0}\right)$ to an arbitrary one $Q_{\varrho}\left(z_{0}\right)$. To this end it suffices to observe that mapping $f(\cdot) \rightarrow \frac{1}{r} f(\dot{\bar{r}})$ is a bijection from $H(r ; \delta, A, \gamma)$ to $H(1 ; \delta, A, \gamma)$ and from $H(r ; A, \gamma)$ to $H(1 ; A, \gamma)$.

It is worth remarking that it seems possible to provide a more constructive proof, based on ideas of Diening and collaborators (see [8] and references therein).

Below is presented proof of Lemma 5.1, which states some algebraic inequalities useful for local estimates of Section 5.

Proof (of Lemma 5.1). Observe first that assumption (2.1d) yields

$$
|A(z, u, q)-A(\tilde{z}, \tilde{u}, q)| \leq C K(2|u|+1)\left(d_{p}(z-\tilde{z})+|u-\tilde{u}|\right)^{\beta}\left(1+|q|^{p-1}\right)
$$

this with $\left|l\left(z_{0}\right)\right|+|\nabla l| \equiv \tilde{M}$ gives

$$
\begin{aligned}
& \left|A(z, u, P)-A\left(z_{0}, l\left(z_{0}\right), P\right)\right| \\
& \quad \leq C K\left(2\left|l\left(z_{0}\right)\right|+1\right)\left(\left|z-z_{0}\right|+\left|u-l\left(z_{0}\right)\right|\right)^{\beta}\left(1+|P|^{p-1}\right) \\
& \quad \leq C(\tilde{M})\left(\varrho^{\beta}+|u-l|^{\beta}+\left|l-l\left(z_{0}\right)\right|^{\beta}\right)\left(1+|P-\mathbb{D} l|^{p-1}+|\mathbb{D} l|^{p-1}\right) \\
& \quad \leq C(\tilde{M})\left(\varrho^{\beta}+|u-l|^{\beta}+\left(|\nabla l|\left|z-z_{0}\right|\right)^{\beta}\right)\left(1+|P-\mathbb{D} l|^{p-1}+\tilde{M}{ }^{p-1}\right) \\
& \quad \leq C(\tilde{M}) \varrho^{\beta}\left[1+|P-\mathbb{D} l|^{p-1}+\left|\frac{u-l}{\varrho}\right|^{p \beta}+|P-\mathbb{D} l|^{p}+\left|\frac{u-l}{\varrho}\right|^{\beta}\right] \\
& \quad \leq C(\tilde{M}) \varrho^{\beta}\left[1+|P-\mathbb{D} l|^{p}+\left|\frac{u-l}{\varrho}\right|^{p}\right]
\end{aligned}
$$

i.e. (5.2a).

Turning to estimate for (5.2b) one has again from (7.34)

$$
\begin{aligned}
& \left|A\left(z_{0}, l\left(z_{0}\right), \mathbb{D} l\right)-A(z, u, \mathbb{D} l)\right| \leq C K\left(\left|l\left(z_{0}\right)\right|\right)\left(\varrho+\left|u-l_{0}\right|\right)^{\beta}(1+|\mathbb{D} l|)^{p-1} \\
& \quad \leq C(\tilde{M})\left[\varrho^{\beta}+|u-l|^{\beta}+\left|l-l\left(z_{0}\right)\right|^{\beta}\right] \leq C(\tilde{M}) \varrho^{\beta}\left[1+\left|\frac{u-l}{\varrho}\right|^{\beta}\right]
\end{aligned}
$$


To show inequality (5.3) write

$$
\begin{aligned}
& |A(z, u, P)-A(z, u, \mathbb{D} l)| \\
& \quad \leq|A(z, u, P)-A(z, l, P)|+|A(z, l, P)-A(z, l, \mathbb{D} l)| \\
& \quad+|A(z, u, \mathbb{D} l)-A(z, l, \mathbb{D} l)| \\
& \quad=I+I I+I I I .
\end{aligned}
$$

Estimate I using (2.1d) and properties of function $K$

$$
\begin{aligned}
& |A(z, u, P)-A(z, l, P)| \leq C \min \left(1, K(|u|+|l|)|u-l|^{\beta}\right)\left(1+|P|^{p-1}\right) \\
& \quad \leq C \min \left(1, K(|u-l|+2|l|)|u-l|^{\beta}\right)\left(1+|P-\mathbb{D} l|^{p-1}+|\mathbb{D} l|^{p-1}\right) \\
& \quad \leq C(\tilde{M}) \min \left(1, K\left(|u-l|+2\left|l-l\left(z_{0}\right)\right|+2 \tilde{M}\right)|u-l|^{\beta}\right)\left(1+|P-\mathbb{D} l|^{p-1}\right)
\end{aligned}
$$

When $|u-l| \geq 1$ we estimate further by

$$
C(\tilde{M})\left(1+|P-\mathbb{D} l|^{p-1}\right) \leq C(\tilde{M})\left(|u-l|+|P-\mathbb{D} l|^{p-1}\right) .
$$

Otherwise by

$$
\begin{aligned}
& C(\tilde{M}) \min \left(1, K(1+2 \varrho|\nabla l|+2 \tilde{M})|u-l|^{\beta}\right)\left(1+|P-\mathbb{D} l|^{p-1}\right) \\
& \quad \leq C(\tilde{M})\left(|u-l|^{\beta}+|P-\mathbb{D} l|^{p-1}\right) .
\end{aligned}
$$

Consequently

$$
I \leq C(\tilde{M})\left(|u-l|^{\beta}+|u-l|+|P-\mathbb{D} l|^{p-1}\right)
$$

Next consider $I I$ in case $|P-\mathbb{D} l| \leq 1$. In view of $|l| \leq \tilde{M}+|\nabla l| \varrho \leq 2 \tilde{M}$ we estimate $I I$ by $(2.2 \mathrm{c})$ as follows

$$
I I=\left|\int_{0}^{1} \frac{\partial A}{\partial q}(z, l, s(P-\mathbb{D} l)+\mathbb{D} l)(P-\mathbb{D} l) d s\right| \leq C_{(2.2 \mathrm{c})}(3 \tilde{M}+1)|P-\mathbb{D} l|
$$

where in case $|P-\mathbb{D} l| \geq 1$ we robustly estimate $I I$ by (2.1c)

$$
\begin{aligned}
I I & \leq C \cdot\left(1+|\mathbb{D} l|^{p-1}+|P|^{p-1}\right) \leq C(\tilde{M})\left(1+|\mathbb{D} l-P|^{p-1}\right) \\
& \leq C(\tilde{M})\left(|\mathbb{D} l-P|+|\mathbb{D} l-P|^{p-1}\right)
\end{aligned}
$$

so from both cases one has

$$
I I \leq C(\tilde{M})\left(|\mathbb{D} l-P|+|\mathbb{D} l-P|^{p-1}\right)
$$


Finally we obtain estimate for III in view of (7.34)

$$
I I I \leq C(\tilde{M})|u-l|^{\beta}
$$

and inequalities for I, I I, I I I give desired (5.3). Inequality (5.4) follows similarly and more straightforwardly, when instead of (7.34) we observe that assumption (2.1d) implies

$$
|A(z, u, q)-A(\tilde{z}, \tilde{u}, q)| \leq C\left(1+|q|^{p-1}\right) .
$$

Acknowledgements. The author is indebted to Petr Kaplický for his interest and remarks. The author thanks Przemek Kamiński for his initial motivation.

Open Access This article is distributed under the terms of the Creative Commons Attribution Noncommercial License which permits any noncommercial use, distribution, and reproduction in any medium, provided the original author(s) and source are credited.

\section{References}

[1] Apushkinskaya, D., Bildhauer, M., Fuchs, M.: Steady states of anisotropic generalized Newtonian fluids. J. Math. Fluid Mech. 7(2), 261-297 (2005)

[2] Beirão da Veiga, H., Crispo, F.: On the global $W^{2, q}$ regularity for nonlinear N-systems of the p-Laplacian type in n space variables. Nonlinear Anal. 75(11), 4346-4354 (2012)

[3] Bojarski, B., Hajłasz, P.: Pointwise inequalities for Sobolev functions and some applications. Studia Math., 106 (1) (1993)

[4] Breit, D., Fuchs, M.: The nonlinear Stokes problem with general potentials having superquadratic growth. J. Math. Fluid Mech. 13(3), 371-385 (2011)

[5] Campanato, S.: Equazioni paraboliche del secondo ordine e spazi $\mathcal{L}^{2, \theta}(\Omega, \delta)$. Ann. Mat. Pura Appl. 73(4), 55-102 (1966)

[6] Desvilettes, L., Villani, C.: On a variant of Korn's inequality arising in statistical medanics. ESAIM Control Optim. Calc. Var. 8, 603-619 (2002)

[7] DiBenedetto, E.: Degenerate parabolic systems. Springer, Berlin (1993)

[8] Diening, L., Stroffolini, B., Verde, A.: The $\phi$-harmonic approximation and the regularity of $\phi$-harmonic maps. J. Differ. Equ. 253(7), 1943-1958 (2012)

[9] Duzaar, F., Mingione, G.: Second order parabolic systems, optimal regularity and singular sets of solutions. AIHP - AN 22, 705-751 (2005)

[10] Duzaar, F., Mingione, G., Steffen, K.: Parabolic Systems with Polynomial Growth and Regularity, Memoirs A.M.S. 214, 2011

[11] Eidelman, S., Zhitarashu, N.: Parabolic Boundary Value Problems, Birkhäuser, 1998

[12] Friedrichs, K.: On the boundary-value problems of the theory of elasticity and Korn's inequality. Ann. of Math. (2) 48, 441-471 (1947)

[13] Kaplický, P., Málek, J., Stará, J.: Global-in-time Hlder continuity of the velocity gradients for fluids with shear-dependent viscosities. NoDEA Nonlinear Differential Equations Appl. 9(2), 175-195 (2002)

[14] Korn, A.: Die Eigenschwingungen eines elastischen Körpers mit ruhender Oberfläche. Akad. der Wiss., München, Math. Phys. Klasse, Ber. 36, 351 (1906)

[15] Kronz, M.: Partial regularity results for minimizers of quasiconvex functionals of higher order. AIHP - AN 19, 81-112 (2002) 
[16] Nečas, J., Málek, J., Ruzička, M., Rokyta, M.: Weak and measure-valued solutions to evolutionary PDEs. Chapman \& Hall (1996)

[17] Prüss, J., Bothe, D.: Lp-theory for a class of non-Newtonian fluids. SIAM J. Math. Anal. 39(2), 379-421 (2007)

[18] Schlag, W.: Schauder and Lp estimates for parabolic systems via Campanato spaces. Comm. P.D.E. 21(7-8), 1141-1175 (1996)

[19] Seregin, G., Ladyzhenskaya, O.: On partial regularity of suitable weak solutions to the three-dimensional Navier-Stokes equations. J. Math. Fluid Mech. 1(4), 356-387 (1999)

[20] Simon, L.: Theorems on regularity and singularity of energy minimizing maps, Lectures in Mathematics ETH Zürich, Birkhäuser Verlag, Basel, 1996

[21] Solonnikov, V.: $L_{p}$ estimates for solutions to the initial boundary-value problem for the generalized Stokes system in a bounded domain. J. Math. Sci. 105(5), 2448$2484(2001)$

[22] Tolksdorf, P.: Everywhere-regularity for some quasilinear systems with a lack of ellipticity. Ann. Mat. Pura Appl. (4) 134, 241-266 (1983)

[23] Uhlenbeck, K.: Regularity for a class of non-linear elliptic systems. Acta Math. 138(34), 219-240 (1977) 